\title{
ACCIONES EQUIVALENTES Y SOLUCIÓN EN DESPLAZAMIENTOS INTERPOLADA EN LA VIGA DE BERNOUILLI-EULER
}

\author{
(EQUIVALENT LOADS AND INTERPOLATED SOLUTION IN DISPLACEMENTS ON \\ BERNOUILLI-EULER'S BEAM)
}

José L. Romero, Dr. Ing. de Caminos. Facultad de Informática, UPM

Miguel A. Ortega, Ing. de Caminos

ESPAÑA

Fecha de recepción: 20-II-98

\begin{abstract}
RESUMEN
Se propone un método para el cálculo de la viga de BernoulliEuler que permite optimizar los resultados obtenidos mediante los elementos finitos hermíticos tradicionales. La principal ventaja es que puede aproximar con gran bondad los desplazamientos y esfuerzos en el interior de los elementos, incluso para elementos de gran tamaño.
\end{abstract}

SUMMARY

A method for the calculus of Bernoulli-Euler's beam is presented. This method allows the results obtained by usual finite element methods to be improve. The main advantage of the proposed method is that it can approximate the displacements, bending moments and shear in the elements, including in large elements, accurately.

\section{Lista de símbolos}

Se recoge aquí el significado de los símbolos más representativos utilizados en este trabajo.

$A=$ función que define la rigidez en cada punto de la viga

$E=$ módulo de elasticidad

$f=$ función que representa la distribución de carga en una viga o elemento

$f^{*}=$ función que representa una distribución de cargas equivalentes af y que produce el mismo vector de cargas nodales equivalentes en cada elemento

$F=$ función que representa una distribución de cargas equivalentes tal que $L F=0$

$\tilde{f}=$ vector de cargas nodales equivalentes en el elemento

$\mathbf{F}=$ vector de cargas nodales equivalentes en la viga

$I=$ momento de inercia 
$\widetilde{K}=$ matriz de rigidez de un elemento

$\mathbf{K}=$ matriz de rigidez global

$L=$ operador diferencial para la ecuación de la viga

$l_{i}, l_{i}^{*}(i=1,2,3,4)=$ formas lineales de interpolación en los extremos de cada elemento

$m=$ función de momentos flectores exactos

$m^{*}=$ función de momentos flectores aproximados con elementos finitos

$M=$ función de momentos flectores aproximados con el método propuesto

$N_{i}(i=1,2,3,4)=$ funciones de forma que son solución de $L N_{i}=0$

$\widetilde{q}=$ vector de cargas nodales de equilibrio en cada elemento

$\mathbf{Q}=$ vector de cargas nodales en la viga

$q=$ función de cortantes exactos

$q^{*}=$ función de cortantes aproximados con el método de elementos finitos

$Q=$ función de cortantes aproximados con el método propuesto

$u=$ solución exacta de la ecuación de la viga $L u=f$

$u^{*}=$ solución aproximada de la ecuación de la viga con el método de elementos finitos

U = solución aproximada de la ecuación de la viga por el método propuesto

\section{Introducción}

El modelo de viga de Bernoulli-Euler, pese a que no tiene en cuenta la influencia del esfuerzo cortante en la deformación de la viga, sigue teniendo vigencia en la mayoría de los cálculos que en la actualidad se realizan con los programas de elementos finitos y cálculo matricial de estructuras. Por otra parte, este sencillo modelo unidimensional permite un cálculo de la solución mediante elementos finitos, con la particularidad de obtener, cuando se emplean determinadas funciones de forma, resultados exactos para desplazamientos y giros en los nodos de los elementos [1], [2], [3], y localizaciones óptimas para la determinación de los esfuerzos dentro del elemento, que dan resultados exactos cuando la carga es constante [4]. En el estudio de elementos contorno se han obtenido resultados análogos para otros problemas unidimensionales [5], [6], y generalizaciones de dichos aspectos relacionados con la exactitud nodal se tratan en [7].

En este artículo se desarrolla particularmente la idea expuesta, en la última referencia sobre soluciones aproximadas, en problemas autoadjuntos, obtenidas mediante interpolación de valores nodalmente exactos y la interpretación de dichas soluciones como soluciones exactas correspondientes a un cierto estado simplificado de la acciones, aplicándose aquí al elemento viga.

El procedimiento consiste en utilizar en primer lugar la solución aproximada obtenida mediante los elementos finitos usuales basados en la interpolación de los dos movimientos en cada nudo extremo del elemento y, posteriormente, realizar una interpolación empleando ocho datos, cuatro de movimientos y otros cuatro relativos a los esfuerzos, estos últimos calculados a partir de la ecuación de equilibrio de cada elemento una vez conocidos los movimientos. Este método permite, en general, emplear elementos finitos de tamaño mayor que el habitual, reduciendo así el volumen de cálculo, pudiendo además realizar un cálculo aproximado de los esfuerzos en el interior del elemento, con resultados considerablemente superiores a los deducidos a partir de la solución aproximada tradicional. 
Por otra parte se destaca el hecho de que la solución aproximada obtenida mediante el tipo de interpolación que se propone, corresponde a una solución exacta para un estado de carga muy particular relacionado con el estado de carga original, permitiendo dicha idea definir estados de carga equivalentes mediante cierto criterio. Este proceso se sistematiza al objeto de determinar cargas repartidas o concentradas equivalentes a otras dadas. Finalmente, hay que indicar que algunos de los aspectos que se exponen se relacionan en parte con diversas técnicas empleadas en la resolución de ecuaciones diferenciales, que se basan en métodos de proyección ortogonal, del término independiente de la ecuación, en espacios de polinomios [8].

\section{Preliminares}

La ecuación de equilibrio del elemento viga puede obtenerse por diversos procedimientos. Aquí se va a obtener integrando la ecuación diferencial, demostrándose posteriormente la relación con la ecuación de equilibrio que resulta a partir de la discretización usual de la formulación débil. La deducción que sigue podría realizarse particularizando resultados de la teoría general de ecuaciones diferenciales ordinarias de orden $2 \mathrm{n}$ autoadjuntas, [9], [10], [11]. Sin embargo, hemos considerado oportuno reproducir, aunque de manera esquemática, los distintos pasos de la formulación general, al ser el orden de la ecuación solamente cuatro. Asimismo, por la orientación de carácter aplicado que se ha querido dar a este trabajo, se ha evitado aquí el explicitar en exceso detalles técnicos sobre el marco funcional en el que se han efectuado los distintos desarrollos.

La ecuación diferencial de la viga a flexión para el caso de rigidez $A(x)$ variable, toma la forma $L u=f(x) \operatorname{con} x \quad(a, b)$ donde el operador diferencial $L$ es $L u=(A(x) u$ ")". Se definen las formas lineales de interpolación de tipo Hermite, relativas a los extremos del elemento $(a, b), l_{i}, i=1, \ldots, 4$, por

$$
l_{1}(u)=u(a), l_{2}(u)=u^{\prime}(a), l_{3}(u)=u(b), l_{4}(u)=u^{\prime}(b)
$$

Sean $N_{i}(x), i=1, . ., 4$ cuatro soluciones linealmente independientes de la ecuación homogénea $L u=0$ en el intervalo $(a, b)$ que verifican $l_{i}\left(N_{j}\right)=\delta_{i j}, i, j=1, . ., 4$. La solución general $L u=f(x)$ puede ponerse como

$$
u=\sum_{i=1}^{4} N_{i} l_{i}(u)+w
$$

donde $w(x)$ es una solución particular de la ecuación completa que verifica

$$
l_{i}(w)=0, \quad i=1, . ., 4
$$

Siempre puede imponerse esta última condición, ya que si es $w^{*}$ una solución particular que toma los valores arbitrarios $l_{i}\left(w^{*}\right)=z_{i}$, la función

$$
w=w^{*}-\sum_{i=1}^{4} N_{i}(x) z_{i}
$$

verifica (3).

Multiplicando ahora por $A(x)$ ambos miembros de (2), después de derivar $u$ dos veces, sustituyendo después en $a$ y $b$. Derivando nuevamente la expresión $A(x) u$ " y sustituyendo posteriormente en los mismos puntos, se obtienen las expresiones de las cargas nodales de equilibrio en los extremos del elemento, es decir, el momento flector y esfuerzo cortante en $x=b$ y los valores opuestos a dichos esfuerzos en $x=a$. Dichas cantidades las expresamos mediante las formas lineales $l_{i}^{*}$, en el orden siguiente

$$
\begin{gathered}
l_{1}^{*}(u)=\left(A(x) u^{\prime \prime}\right)_{x=a}^{\prime}, l_{2}^{*}(u)=-\left(A(x) u^{\prime \prime}\right)_{x=a}, \\
l_{3}^{*}(u)=-\left(A(x) u^{\prime \prime}\right)_{x=b}^{\prime}, l_{4}^{*}(u)=\left(A(x) u^{\prime \prime}\right)_{x=b}
\end{gathered}
$$

pudiendo ponerse la siguiente expresión matricial, denominada ecuación de equilibrio local o ecuación de equilibrio del elemento, la cual es exacta por construcción

$$
\tilde{K} \tilde{u}=\tilde{f}+\tilde{q}
$$


donde, tal y como se demostrará después, $\widetilde{K}$ es la matriz de rigidez del elemento viga, $\widetilde{u}$ el vector de desplazamientos nodales del elemento, $\widetilde{f}$ el vector de cargas nodales equivalentes del elemento y $\widetilde{q}$ el de cargas nodales de equilibrio del elemento, con

$$
\begin{gathered}
\tilde{f}^{t}=\left(-l_{1}^{*}(w),-l_{2}^{*}(w),-l_{3}^{*}(w),-l_{4}^{*}(w)\right), \quad \tilde{q}^{t}=\left(l_{1}^{*}(u), l_{2}^{*}(u), l_{3}^{*}(u), l_{4}^{*}(u)\right) \\
\tilde{u}^{t}=\left(u(a), u^{\prime}(a), u(b), u^{\prime}(b)\right)
\end{gathered}
$$

y la matriz $\widetilde{K}=\left(k_{i j}\right)_{i, j=1, \ldots 4}$ donde $k_{i j}=l_{i}^{*}\left(N_{j}\right)$

(I) El primer vector del segundo miembro de (6) es el vector de cargas nodales equivalentes, es decir

$$
\int_{a}^{b} f N_{j} d x=-l_{j}^{*}(w), j=1, . .4 .
$$

En efecto, integrando por partes

$$
\int_{a}^{b} f N_{j} d x=\int_{a}^{b}\left(A w^{\prime \prime}\right)^{\prime \prime} N_{j} d x=\sum_{i=1}^{4}\left(l_{i}\left(N_{j}\right)\left(-l_{i}^{*}(w)+l_{i}^{*}\left(N_{j}\right) l_{i}(w)\right)+\int_{a}^{b}\left(A N_{j}^{\prime \prime}\right)^{\prime \prime} d x=-l_{j}^{*}(w)\right.
$$

Obsérvese que $\quad l_{i}\left(N_{j}\right)=\delta_{i j}, \quad l_{i}(w)=0, i=1, . .4, \quad\left(A N_{j}^{\prime \prime}\right)^{\prime \prime}=0$

(II) La matriz $\widetilde{K}$ es la misma matriz de rigidez que se obtiene en la forma clásica a partir de la discretización de la formulación débil, es decir

$$
k_{i j}=\int_{a}^{b} A N_{i}^{\prime \prime} N_{j}^{\prime \prime} d x
$$

para funciones de forma tales que $L N_{i}=L N_{j}=0$.

En efecto, integrando por partes el producto $(A g$ ") " $h$ para dos funciones arbitrarias, se tiene

$$
\int_{a}^{b}\left(A g^{\prime \prime}\right)^{\prime \prime} h d x=\sum_{i=1}^{4} l_{i}(h)\left(-l_{i}^{*}(g)\right)+\int_{a}^{b} A g^{\prime \prime} h^{\prime \prime} d x
$$

que aplicado a las funciones de forma $N_{i} y N_{j}$ da lugar a

$$
\begin{aligned}
k_{i j}=k_{j i}=\int_{a}^{b} A N_{i}^{\prime \prime} N_{j}^{\prime \prime} d x & =\int_{a}^{b}\left(A N_{i}^{\prime \prime}\right)^{\prime \prime} d x \\
& -\sum_{p=1}^{4} l_{p}\left(N_{j}\right)\left(-l_{p}^{*}\left(N_{i}\right)\right)=l_{j}^{*}\left(N_{i}\right)=l_{i}^{*}\left(N_{j}\right)
\end{aligned}
$$

La construcción de la matriz $\widetilde{K}$ a partir del resultado $k_{i j}=l_{i}{ }^{*}\left(N_{j}\right)$ para el caso particular de rigidez $A(x)=E I$ constante, es inmediata. En efecto, las funciones de forma para este caso son

$$
\begin{aligned}
& N_{1}(x)=1-3 x^{2} / l^{2}+2 x^{3} / l^{3}, N_{2}(x)=x-2 x^{2} / l+x^{3} / l, \quad N_{3}(x)=3 x^{2} / l^{2}-2 x^{3} / l^{3} \\
& N_{4}(x)=-x^{2} / l+x^{3} / l^{2}, x \in(a, b), l=b-a
\end{aligned}
$$

resultando la clásica matriz

$$
\tilde{K}=\frac{E I}{l^{3}}\left[\begin{array}{cccc}
12 & 6 l & -12 & 6 l \\
6 l & 4 l^{2} & -6 l & 2 l^{2} \\
-12 & -6 l & 12 & -6 l \\
6 l & 2 l^{2} & -6 l & 4 l^{2}
\end{array}\right]
$$

Debe observarse cómo trabajando directamente con la solución completa de la ecuación diferencial de la viga, se ha llegado al mismo resultado, sobre la matriz de rigidez y los vectores de cargas nodales del elemento, que a partir de la discretización usual de la formulación débil o expresión de los desplazamientos virtuales 


$$
\int_{a}^{b} A u^{\prime \prime} v^{\prime \prime} d x=\int_{a}^{b} f v d x+\sum_{i=1}^{4} l_{i}(v) l_{i}^{*}(u)
$$

(III) Considerando la viga definida en el intervalo de extremos $x_{1}, x_{n}$ descompuesta en la forma

$$
\left[x_{1}, x_{n}\right]=\bigcup_{i=1}^{n-1}\left[x_{i}, x_{i+1}\right]
$$

donde cada elemento $\left[x_{i}, x_{i+1}\right]$ es un caso particular del elemento genérico de extremos a y $b$, los desplazamientos en los nodos $x_{i}$ vienen dados por los valores exactos $u\left(x_{i}\right)$ y $u^{\prime}\left(x_{i}\right), i=1, . ., n$ que se obtienen de la ecuación de la ecuación de equilibrio global

$$
\mathbf{K U}=\mathbf{F}+\mathbf{Q}
$$

una vez consideradas las condiciones de vínculo.

En efecto, los resultados anteriores permiten poner de manifiesto que la ecuación de equilibrio global (13) que se obtiene después de ensamblar en la forma usual, las ecuaciones del tipo (6) relativas a cada elemento, es la misma que resulta de la discretización de la formulación débil cuando se emplean funciones de forma que son solución de la homogénea. Por otro lado, de la ecuación de equilibrio global, una vez consideradas aquellas condiciones de contorno que imposibilitan el movimiento de la viga como sólido rígido, es decir las que impiden los desplazamientos definidos por polinomios de primer grado, se obtiene solución única para los desplazamientos desconocidos en los nodos. Como dichos desplazamientos son verificados por la solución exacta por la igualdad de ecuaciones de equilibrio, resulta que los desplazamientos nodales obtenidos son exactos.

En las referencias [1] y [7] se analizan con cierto detalle las propiedades de las soluciones aproximadas obtenidas a partir de la formulación débil de problemas autoadjuntos $L(u)=f$ cuando la solución dentro del elemento se aproxima con funciones del espacio de soluciones de la ecuación diferencial homogénea. Finalmente, una vez obtenidos los desplazamientos exactos de cada nodo, entrando en la ecuación de equilibrio de cada elemento pueden obtenerse las cargas nodales de equilibrio en los extremos del elemento, las cuales son exactas también en el caso particular que nos ocupa debido a que las ecuaciones de equilibrio de cada elemento son también exactas.

\section{Acciones equivalentes y solución en desplazamientos interpolada}

A partir del cálculo del vector de cargas nodales en $(a, b)$ puede observarse que hay infinitos estados de carga correspondientes a cada ecuación de equilibrio que proporcionan las mismas cargas nodales, pues como puede verse la función $f$ interviene solamente con los cuatro valores

$$
\left(f, N_{i}\right)=\int_{a}^{b} f N_{i} d x, i=1, . ., 4
$$

donde $\left(f, N_{i}\right)$ designa el producto escalar usual extendido al espacio de distribuciones o funciones generalizadas [11], [12].

NOTA: Consideramos solamente distribuciones, $f$, dadas por funciones continuas a trozos para representar acciones repartidas y deltas de Dirac y dipolos para acciones puntuales. De esta manera la solución será, al menos, de la clase $\mathrm{C}^{1}$ para el caso más frecuente de rigidez $A(x)$ continua a trozos en todo el dominio, con $A(x)$ de la clase $\mathrm{C}^{2}$ en cada elemento (a,b), cumpliendo además la condición $A(x) \geq A>0$, con esto las soluciones de la ecuación homogénea $L u=0$ son de la clase $\mathrm{C}^{4}$. Asimismo las derivadas de $u$, de orden dos o superior, que aparecen en los desarrollos que siguen, deben entenderse en el sentido de las distribuciones.

Véase que para dos acciones distintas $f$ y $f^{*}$ en $(a, b)$ se verifica

$$
\left(f, N_{i}\right)=\left(f^{*}, N_{i}\right), \quad i=1, . ., 4
$$

cuando $f$ - $f^{*}$ es ortogonal a cada una de las funciones de forma $N_{i} i=1, . ., 4$ o lo que es equivalente, a los elementos del subespacio $N(L)$ (núcleo del operador diferencial $L$ ).

De lo anterior se tiene el siguiente resultado: 
(IV) Dada la acción o estado de carga fen $(a, b)$ existe otro estado perteneciente al espacio de soluciones de la ecuación homogénea, que genera las mismas cargas nodales equivalentes que $f$, siendo $F$ la proyección ortogonal de $f$ en el citado espacio. Además, la solución $U$ de la ecuación $L U=F$ que satisface $l_{i}(u)=l_{i}(U), i=, . ., 4$ verifica, consecuentemente, $l_{i}^{*}(u)=l_{i}^{*}(U) \cdot U$ es, por tanto, la solución de $L^{2} U=0$ que interpola los valores $l_{i}(u)$ y $l_{i}^{*}(u), i=1, \ldots, 4$.

Lo anterior es análogo a decir que $U$ interpola los valores $u, u$ ', $u$ ', $u$ ', ' en $a$ y $b$ tal y como puede demostrarse trivialmente. En la práctica no es necesario, en consecuencia, calcular $F$ para obtener $U$, pues basta obtener el elemento del núcleo de $L^{2}$ que interpola los ocho valores indicados.

NOTA: Para asegurar suficiente regularidad a los elementos del núcleo de $L^{2}, A(x)$ debería ser al menos de la clase $\mathrm{C}^{6}$ dentro de cada intervalo $(a, b)$. Así $U$ y sus derivadas, hasta el orden ocho, serían funciones en el sentido clásico.

Por otra parte, si se desea obtener la expresión de la carga $F$ sin conocer $U$ basta aplicar el teorema de la proyección ortogonal, teniéndose

$$
F=\left(N_{1}, N_{2}, N_{3}, N_{4}\right) G^{-1}\left(\begin{array}{l}
\left(f, N_{1}\right) \\
\left(f, N_{2}\right) \\
\left(f, N_{3}\right) \\
\left(f, N_{4}\right)
\end{array}\right)
$$

donde $G=\left(g_{i j}\right)_{i, j=1, \ldots, 4}$ con $g_{i j}=\left(N_{i}, N_{j}\right)$. Asimismo, si $U$ se ha determinado mediante interpolación, entonces $F$ puede determinarse a partir de $F=L U$. Obsérvese que como consecuencia de ser $F$ combinación lineal de las $N_{i}, i=1,2,3,4$, dicha acción es una función continua de la clase $\mathrm{C}^{4}$ en el elemento, es decir es una acción repartida con gran regularidad.

La equivalencia que se define entre dos estados de carga en el elemento $(a, b)$ es $f=f^{*}$ si $\left(f-f^{*}, N_{i}\right)=0, i=1, . ., 4$ y $F$ es, precisamente, un cierto estado simplificado de cargas asociado a cada clase, es decir, un representante canónico de la clase de equivalencia.

Es interesante destacar, como consecuencia inmediata de lo anterior, que aquellos estados de carga $f$ para los que se tenga la ortogonalidad $\left(f, N_{i}\right)=0, i=1, . ., 4$ se verifica que si los desplazamientos nodales son nulos entonces las cargas nodales de equilibrio son nulas. Por ejemplo, en el caso de rigidez constante, $A(x)=E I$, aquellas cargas repartidas $f$ que vengan dadas por un polinomio ortogonal de Legendre, en el elemento $(a, b)$, de grado igual o superior a cuatro, no originan ningún esfuerzo en los extremos del elemento viga cuando la viga está biempotrada, es decir cuando $u^{t}=(0,0,0,0)$. Para estos casos la solución aproximada obtenida mediante interpolación de los valores $l_{i}(u)=l_{i}^{*}(u)=0, i=1, . ., 4$ es $U=0$.

Por otra parte, llamando $m(x)=A(x)$ u' y $q(x)=-(A(x)$ u') ' al momento flector y esfuerzo cortante para la solución exacta y $M(x)=A(x) U^{\prime \prime y} Q(x)=-\left(A(x) U^{\prime \prime}\right)$ ' a los mismos esfuerzos para la solución aproximada, resulta además que

(V) Para cada elemento $(a, b)$ se tienen las siguientes ortogonalidades

$$
\left(m(x)-M(x), N_{i}^{\prime}\right)=0, \quad\left(q(x)-Q(x), N_{i}^{\prime}\right)=0, \quad i=1, . ., 4
$$
En efecto, teniendo en cuenta que $\int_{a}^{b} f N_{i} d x=\int_{a}^{b} F N_{i} d x, i=1, . ., 4$ resulta, integrando por partes y observando que
$q(a)=Q(a)$ y $q(b)=Q(b)$

$$
0=\int_{a}^{b}\left(\left(A(x) u^{\prime \prime}\right)^{\prime \prime}-\left(A(x) U^{\prime \prime}\right)^{\prime \prime}\right) N_{i} d x=\int_{a}^{b}(q(x)-Q(x)) N_{i}^{\prime} d x, i=1, . ., 4
$$

Análogamente, integrando por partes nuevamente y considerando que $m(a)=M(a)$ y $m(b)=M(b)$ resulta

$$
0=\int_{a}^{b}(q(x)-Q(x)) N_{i}^{\prime} d x=\int_{a}^{b}(m(x)-M(x)) N_{i}^{\prime \prime} d x, i=1, . ., 4
$$

Teniendo en cuenta ahora los espacios de funciones engendrados por $N_{i}^{\prime}$ y $N_{i}$ ' para $i=1, . ., 4$ el resultado anterior puede expresarse de esta otra forma 
(VI) La diferencia de momentos $m(x)$ - $M(x)$ en (a,b) es ortogonal al espacio de funciones, de dimensión dos, engendrado por la base de funciones $B_{m}=1 / A(x), x / A(x)$. Asimismo, la diferencia de esfuerzos cortantes es ortogonal al espacio de funciones, de dimensión tres, engendrado por la base

$$
B_{q}=\left\{1, \int_{a}^{x}(1 / A(t)) d t, \int_{a}^{x}(t / A(t)) d t\right\}
$$

En efecto, llamando $v(x)$ al elemento genérico engendrado por las funciones $N_{i}, 1, \ldots, 4$, dicho elemento es solución de $\left(A(x) v^{\prime \prime}\right)$ "' $=0$ y recíprocamente. Pero

$$
v^{\prime \prime}=c_{1}(1 / A(x))+c_{2}(x / A(x)) \text { y } v^{\prime}=c_{1} \int_{a}^{x}(1 / A(t)) d t+c_{2} \int_{a}^{x}(t / A(t)) d t+c_{3}
$$

con $c_{1}, c_{2}$ y $c_{3}$ arbitrarias, luego los espacios engendrados por $N_{i}^{\prime \prime}, i=1, \ldots, 4$ y $N_{i}^{\prime}=1, \ldots, 4$ son los engendrados respectivamente por las bases de funciones indicadas.

Obsérvese que además de las ortogonalidades anteriores se tiene que el momento flector aproximado, $M(x)$, interpola en el sentido clásico de Hermite, al momento flector exacto, $m(x)$, en los extremos del elemento, es decir

$$
M(a)=m(a), M^{\prime}(a)=m^{\prime}(a), M(b)=m(b) \text { y } M^{\prime}(b)=m^{\prime}(b)
$$

Análogamente, el esfuerzo cortante aproximado $Q(x)$ interpola al exacto $q(x)$ solamente en el sentido de Lagrange $Q(a)=q(a)$ y $Q(b)=q(b)$ aunque la diferencia entre ambos es, en este caso, ortogonal a un espacio de funciones de dimensión tres.

Debe tenerse en cuenta en lo anterior que el momento flector exacto $m(x)$ es, para las acciones que venimos considerando, una función discontinua en general dentro del elemento y, en consecuencia, el cortante exacto $q(x)$, contiene en los puntos donde hay saltos en el momento, a las distribuciones dadas por las deltas de Dirac.

Los resultados anteriores son relativos a cada elemento genérico $(a, b)$, pero se extienden de manera inmediata a todo el dominio. En efecto, la equivalencia de acciones en todo el dominio puede definirse del siguiente modo: dos acciones son equivalentes en todo el dominio si restringidas a cada elemento resultan equivalentes. Por otra parte si una acción se sustituye por otra equivalente en todo el dominio, es decir, si $f$ se sustituye dentro de cada elemento $\left[x_{i}, x_{i+}\right], i=1, \ldots, n-1$, por cualquier otra $f^{*}$ equivalente, las ecuaciones de equilibrio local no se modifican $\mathrm{y}$, consecuentemente, la ecuación de equilibrio global (13) es la misma para todas las acciones equivalentes. Se concluye de manera inmediata de lo anterior que, los desplazamientos en los nodos $x_{i}, i=\underline{l}, . ., n$ para $f \mathrm{y} f^{*}$ son los mismos cuando dichas acciones son equivalentes.

Por otra parte, respecto a la regularidad de la solución aproximada $U(x)$ puede indicarse que es $\mathrm{C}^{1}$ en todo el dominio, cuando hay cargas puntuales y momentos en los nodos de separación de elementos consecutivos, independientemente de cómo sea la rigidez $A(x)$. Sin embargo, cuando dichas acciones son nulas en los nodos y $A(x)$ es globalmente al menos de la clase $\mathrm{C}^{1}$, la solución es globalmente, al menos de la clase $\mathrm{C}^{3}$, tal y como se deduce de la nulidad de las componentes correspondientes del vector $\boldsymbol{Q}$ de acciones nodales de (13) donde

$$
\text { componente i de } \mathbf{Q}^{t}=\left(-\left(A u^{\prime \prime}\right)^{\prime}\left(x_{i}^{-}\right)+\left(A u^{\prime \prime}\right)^{\prime}\left(x_{i}^{+}\right),\left(A u^{\prime \prime}\right)\left(x_{i}^{-}\right)-\left(A u^{\prime \prime}\right)\left(x_{i}^{+}\right)\right)
$$

En general, para lo que sigue, el análisis se efectuará, con objeto de simplificar los desarrollos, a nivel de elemento en lugar de hacerlo para todo el dominio, ya que la extensión de los resultados a todo el dominio es inmediata.

Es interesante finalizar este punto comparando los resultados obtenidos con los que se derivan de la solución aproximada usual, $u^{*}(x)$, es decir, la que se obtiene a partir de la interpolación, únicamente, de los movimientos, $u(a), u^{\prime}(a), u(b), u^{\prime}\left(b^{\prime}\right)$, de los extremos de cada elemento, o sea

$$
u^{*}=\sum_{i=1}^{4} N_{i} l_{i}(u)
$$

Sea $m^{*}(x)=A(x) u^{*}$ "' $(x)$ el momento aproximado. Se tiene, a partir de la formulación débil, la ortogonalidad siguiente

$$
\int_{a}^{b}\left(A(x) u^{\prime \prime}-A(x) u^{* \prime}\right) N_{i}^{\prime \prime} d x=0, i=1, . ., 4
$$

es decir, la diferencia de momentos, $m(x)-m^{*}(x)$, es ortogonal al espacio de funciones engendrado por las derivadas segundas de las funciones de forma. Según los resultados del punto (VI), dicha diferencia de momentos es ortogonal al 
espacio de funciones engendrado por la base $B_{m}$. Este resultado es análogo al del método propuesto en cuanto a ortogonalidad se refiere, sin embargo nuestro resultado verifica además la propiedad de interpolación, pues los momentos interpolan en el sentido de Hermite tal y como se indica en (18).

Por otra parte, para el cortante aproximado $q^{*}(x)=-\left(A(x) u^{* \prime \prime}\right)$ ' se obtiene, integrando por partes (19), el siguiente resultado

$$
\left.N_{i}^{\prime}\left(m(x)-m^{*}(x)\right)\right|_{a} ^{b}+\int_{a}^{b}\left(q(x)-q^{*}(x)\right) N_{i}^{\prime} d x=0
$$

es decir, la diferencia de esfuerzos cortantes es ortogonal al espacio de funciones engendrado únicamente por $N_{1}{ }^{\prime}$ y $N_{3}{ }^{\prime}$, pues de las funciones derivadas de las cuatro funciones de forma relativas al elemento, solamente las funciones indicadas se anulan simultáneamente en los extremos del elemento y, en consecuencia, también se anula el primer sumando de (20). Pero el espacio engendrado por dichas funciones es sólo de dimensión uno, tal y como puede demostrarse al ser $N_{1}+N_{3}=1 \mathrm{y}$, por tanto, $N_{1}{ }^{\prime}=N_{3}{ }^{\prime}$. En consecuencia, este resultado difiere notablemente del que se consigue por el método propuesto en el que, además de ser la diferencia de cortantes ortogonal al espacio de dimensión tres engendrado por la base $B_{q}$, el cortante aproximado interpola al exacto, en el sentido de Lagrange, en los extremos del elemento.

Aunque, en lo que a aplicaciones se refiere, en este trabajo, vamos a desarrollar la determinación de la carga repartida $F(x)$ y la correspondiente solución en desplazamientos aproximada, $U(x)$, indicamos a continuación la posibilidad de obtener acciones concentradas equivalentes en el interior de cada elemento. Esta cuestión puede tener interés en ciertas aplicaciones donde interese sustituir acciones repartidas por acciones concentradas en ciertos puntos, y determinar dichas acciones concentradas de una manera sistemática.

Se trata de determinar el valor de las cargas puntuales $P_{1}, P_{2}, \ldots, P_{k}$, situadas en los puntos conocidos $c_{1}, c_{2}, \ldots, c_{k} \in(a, b)$, de tal manera que el nuevo estado de carga que puede definirse mediante deltas de Dirac en la forma

$$
f *(x)=\sum_{i=1}^{k} P_{i} \delta\left(x-c_{i}\right)
$$

sea equivalente al dado por la acción $f$. Se plantea, en consecuencia, el siguiente sistema de cuatro ecuaciones y k incógnitas

$$
\left\{\begin{array}{l}
\sum_{i=1}^{k} N_{j}\left(c_{i}\right) P_{i}=\left(f, N_{j}\right) \\
j=1,2,3,4
\end{array}\right.
$$

El sistema anterior tiene solución única para $\mathrm{k}=4$ pues la matriz de elementos $N_{j}\left(c_{i}\right)$ es regular al ser el conjunto de funciones $N_{j}, j=1,2,3,4$ un sistema de Tschebyscheff [13].

Para el caso particular de rigidez constante y elegir $c_{i}, i=1,2,3,4$ igual a las cuatro raíces del polinomio ortogonal de Legendre de grado cuatro en el elemento $(a, b)$, no es necesario resolver (22) para determinar las cargas $P_{i}$, pues, en este caso, dichas cargas vienen dadas por el producto de los pesos $W_{i}$ de la fórmula de cuadratura de Gauss-Legendre de cuatro puntos $\left(\operatorname{los} c_{i}\right.$ ) por los valores de la función $F(x)$ en dichos puntos. En efecto, la proyección ortogonal $F(x)$ de la carga $f$, es un polinomio de grado tres y como las $N_{j}$ son también polinomios de grado tres, se tiene, empleando la cuadratura citada que es exacta para polinomios de grado siete, la siguiente cadena de igualdades

$$
\left(f, N_{j}\right)=\sum_{i=1}^{4} P_{i} N_{j}\left(c_{i}\right)=\left(F, N_{j}\right)=\int_{a}^{b} F(x) N_{j}(x) d x=\sum_{i=1}^{4} W_{i} F\left(c_{i}\right) N_{j}\left(c_{i}\right)
$$

En consecuencia $P_{i}=W_{i} F\left(c_{i}\right), i=1,2,3,4$.

También es posible determinar una acción $f^{*}$ equivalente a la acción de partida $f$, formada por cargas puntuales y momentos. Limitándonos para simplificar, por ejemplo, al caso de $f^{*}$ dada por dos cargas puntuales y dos momentos, es decir

$$
f^{*}(x)=P_{1} \delta\left(x-c_{1}\right)-M_{1} \delta^{\prime}\left(x-c_{2}\right)+P_{2} \delta\left(x-c_{3}\right)-M_{2} \delta^{\prime}\left(x-c_{4}\right)
$$

La determinación de las cargas y momentos se reduce a la resolución del siguiente sistema 


$$
\left[\begin{array}{llll}
N_{1}\left(c_{1}\right) & N_{1}^{\prime}\left(c_{2}\right) & N_{1}\left(c_{3}\right) & N_{1}^{\prime}\left(c_{4}\right) \\
N_{2}\left(c_{1}\right) & N_{2}^{\prime}\left(c_{2}\right) & N_{2}\left(c_{3}\right) & N_{2}^{\prime}\left(c_{4}\right) \\
N_{3}\left(c_{1}\right) & N_{3}^{\prime}\left(c_{2}\right) & N_{3}\left(c_{3}\right) & N_{3}^{\prime}\left(c_{4}\right) \\
N_{4}\left(c_{1}\right) & N_{4}^{\prime}\left(c_{2}\right) & N_{4}\left(c_{3}\right) & N_{4}^{\prime}\left(c_{4}\right)
\end{array}\right]\left[\begin{array}{c}
P_{1} \\
M_{1} \\
P_{2} \\
M_{2}
\end{array}\right]=\left[\begin{array}{c}
\left(f, N_{1}\right) \\
\left(f, N_{2}\right) \\
\left(f, N_{3}\right) \\
\left(f, N_{4}\right)
\end{array}\right]
$$

Obsérvese que para el caso $c_{1}=c_{2}=a, c_{3}=c_{4}=b$ la matriz de coeficientes del sistema es la idéntica y la cargas equivalentes obtenidas son las nodales equivalentes usuales.

Lo anterior permite establecer una simetría total en el proceso de tratamiento de las acciones, pues como se ha indicado, las acciones repartidas pueden transformarse en otras equivalentes pero concentradas y situadas en puntos arbitrarios de cada elemento. Asimismo, acciones concentradas pueden transformarse en otras repartidas. Esto último permitiría transformar en cargas repartidas, acciones puntuales aplicadas incluso en los nodos de separación de elementos consecutivos. No obstante esta situación que es muy interesante por regularizar la carga en todo el dominio, al convertir la acción exterior en una carga distribuida a lo largo de toda la longitud de la viga, no la consideraremos en este trabajo, ya que dichas cargas en los nodos de separación solamente se tendrán en cuenta en el proceso de ensamblado formando parte del vector de cargas nodales de equilibrio.

\section{Desplazamientos y esfuerzos interpolados en el caso de rigidez constante}

La determinación de $U$ puede entrañar cierta dificultad para el caso donde la expresión de la función $A(x)$ que define la rigidez en cada elemento no sea suficientemente sencilla. En este sentido la utilización de herramientas de cálculo simbólico como Mathematica o Maple, puede facilitar dicha labor. Sin embargo,para el caso de rigidez $A(x)=E I$ constante en cada elemento $(a, b)$, la solución aproximada $U$ correspondiente al estado simplificado de carga $F$, viene dada, sencillamente, por el polinomio de interpolación de Hermite de grado siete que interpola los valores $\boldsymbol{u}^{(i)}(a), u^{(i)}(b), i=0,1,2,3$.

La expresión de dicho polinomio en el intervalo $(a, b)$ es

$$
U(x)=\hat{u} C \hat{x}
$$

donde

$$
\begin{aligned}
& \hat{u}=\left(u(a), u^{\prime}(a), u^{\prime \prime}(a), u^{\prime \prime}(a), u(b), u^{\prime}(b), u^{\prime \prime}(b), u^{\prime \prime}(b)\right) \\
& h=b-a \\
& \hat{x}^{t}=\left(1, z^{1}, z^{2}, z^{3}, z^{4}, z^{5}, z^{6}, z^{7}\right), \quad z=x-a \\
& C=\left(\begin{array}{cccccccc}
1 & 0 & 0 & 0 & -35 / h^{4} & 84 / h^{5} & -70 / h^{6} & 20 / h^{7} \\
0 & 1 & 0 & 0 & -20 / h^{3} & 45 / h^{4} & -36 / h^{5} & 10 / h^{6} \\
0 & 0 & 1 / 2 & 0 & -5 / h^{2} & 10 / h^{3} & -15 /\left(2 h^{4}\right) & 2 / h^{5} \\
0 & 0 & 0 & 1 / 6 & -2 /(3 h) & 1 / h^{2} & -2 /\left(3 h^{3}\right) & 1 /\left(6 h^{4}\right) \\
0 & 0 & 0 & 0 & 35 / h^{4} & -84 / h^{5} & 70 / h^{6} & -20 / h^{7} \\
0 & 0 & 0 & 0 & -15 / h^{3} & 39 / h^{4} & -34 / h^{5} & 10 / h^{6} \\
0 & 0 & 0 & 0 & 5 /\left(2 h^{2}\right) & -7 / h^{3} & 13 /\left(2 h^{4}\right) & -2 / h^{5} \\
0 & 0 & 0 & 0 & -1 /(6 h) & 1 /\left(2 h^{2}\right) & -1 /\left(2 h^{3}\right) & 1 /\left(6 h^{4}\right)
\end{array}\right)
\end{aligned}
$$

Para este caso particular y en el supuesto que la acción $f$ tenga, en cada elemento $(a, b)$, suficiente regularidad (al menos que sea de la clase $\mathrm{C}^{4}$ ) el error $e(x)$ de la solución aproximada $U(x)$ del método propuesto, viene dado [14], por

$$
|e(x)|=|u(x)-U(x)| \leq\left\{\operatorname{máx}\left|f^{4}(t)\right| / E I, t \in[a, b]\right\} x-\left.a\right|^{4}|x-b|^{4} / 8 !
$$


No obstante, el interés práctico de la expresión anterior es pequeño debido a que en la mayor parte de los casos $f$ es una distribución que vendrá dada, dentro del elemento, por funciones continuas a trozos (cargas repartidas), deltas de Dirac (cargas puntuales) y dipolos o derivadas de deltas (momentos).

La expresión del momento aproximado $M(x)=A(x) U^{\prime \prime}=E I U^{\prime \prime}$ 'viene dada por un polinomio de grado cinco y la del cortante $Q(x)=-\left(A(x) U^{\prime \prime}\right)$ ' $=-E I U^{\prime \prime \prime}$ por un polinomio de grado cuatro. En este caso las aproximaciones del desplazamiento y de los esfuerzos resultan exactas para cargas $f(x)$ dadas por un polinomio de grado menor o igual que tres.

Obsérvese que cuando se emplea la solución aproximada usual, los momentos deducidos de dicha solución, para una carga repartida constante, solamente son exactos en los dos puntos de Gauss, es decir en los correspondientes a las raíces del polinomio de Legendre de grado dos en el intervalo $(a, b)$ tal y como se expone en [4] o se deduce, de manera inmediata, de (19) a partir de lo que se indica a continuación. En efecto, cuando $f$ es constante, la solución exacta es un polinomio de cuarto grado y la aproximada usual un polinomio de grado tres, el momento exacto es un polinomio de grado dos, y el momento aproximado un polinomio de grado uno, en consecuencia (19) representa la ortogonalidad de un polinomio de grado dos (que es la diferencia entre el momento exacto y el aproximado), con cualquier polinomio de primer grado, por tanto dicha diferencia es el polinomio ortogonal de Legendre de grado dos. Lo anterior indica también que el momento aproximado es la proyección ortogonal del exacto en el espacio de los polinomios de primer grado.

Por otro lado, por lo que se refiere al cortante para la solución aproximada usual en el caso de carga repartida constante, el cortante exacto viene dado por un polinomio de grado uno y el cortante aproximado por una constante, siendo la diferencia un polinomio de primer grado que es ortogonal al polinomio de segundo grado $N_{1}$ ' el cual es simétrico respecto al centro del elemento, obsérvese que dicho polinomio se anula en los extremos del elemento. De lo anterior se deduce de manera sencilla que el cortante exacto y el aproximado coinciden únicamente en el centro del elemento.

Los resultados anteriores relativos a la bondad de la solución clásica por elementos finitos, aun siendo importantes, quedan muy limitados, comparativamente, en relación con los que se obtienen por el método propuesto, donde para carga no sólo constante sino para cualquier carga que venga dada por un polinomio de grado menor o igual que tres dentro de cada elemento, el desplazamiento, el giro, el momento flector y el esfuerzo cortante, resultan exactos en todo punto del elemento.

\section{Aplicaciones}

Se desarrollan a continuación dos ejemplos donde se aplica el método propuesto. En el primero se trata el caso de una viga biempotrada con rigidez constante en toda su longitud. Aquí se ha considerado sólo un elemento. Sin embargo, para el segundo caso, donde la rigidez es diferente para tres tramos distintos y constante en cada uno de ellos, se ha ido también al menor número de elementos, definiendo un único elemento por tramo. En ambos ejemplos se compara la solución aproximada que da el método propuesto con la solución exacta y con la solución aproximada que da el método de elementos finitos, tanto para la deformada como para las leyes de momentos y cortantes.

\subsection{Ejemplo 1}

Para los tres casos que se indican en la figura 1 sólo se ha considerado un elemento y como la viga es biempotrada, son nulos los desplazamientos y giros en los extremos. Resulta de lo anterior que el primer miembro de (6) es nulo y $\widetilde{q}=-\widetilde{f}$, donde las componentes de $\widetilde{f}$ se calculan mediante $\left(f, N_{i}\right)$. Se obtienen entonces los valores $u$ ', $u$ ', 'en los extremos del elemento. Con los ocho valores $u^{(i)}(a), u^{(i)}(b), i=0,1,2,3$ se obtiene mediante (26) la solución aproximada $U(x)$ que denominamos solución por el método propuesto. De dicha solución se obtienen el momento y cortante aproximados $M(x)=A(x) U^{\prime \prime}, Q(x)$ $=-\left(A(x) U^{\prime \prime}\right)$ 'respectivamente. Si se quiere puede obtenerse después la acción repartida equivalente $F(x)=\left(A(x) U^{\prime \prime}\right)$ ',. Estas operaciones resultan triviales cuando la rigidez es constante, como ocurre en el presente caso.

Para los ejemplos considerados debe observarse que la solución por elementos finitos tradicional $u^{*}(x)$ es idénticamente nula, así como el momento flector y el cortante deducidos de ella. Sin embargo, como puede apreciarse en los apartados (b), (c) y (d) de las figuras 2,3 y 4, las leyes de deformada, flectores y cortantes exactos y las obtenidas por el método propuesto son considerablemente próximas entre sí, especialmente para la carga puntual, figura 2, y para la carga repartida en parte del vano, figura 3.

Por otra parte, en el caso del momento puntual, figura 4, la aproximación es más deficiente. Obsérvese que para los casos considerados en este ejemplo, la falta de regularidad de la acción $f$ es notable e, incluso así, las aproximaciones obtenidas 
para las diferentes leyes son muy aceptables. En el caso de mayor regularidad de la acción, que es el de la carga repartida en parte del vano, las aproximaciones, sin entrar en mayores precisiones, pueden calificarse de muy notables.

La razón fundamental de la bondad de dichas aproximaciones, especialmente las referentes a las leyes de momentos flectores y esfuerzos cortantes, la atribuimos a las propiedades de ortogonalidad citadas en (V) y (VI) y, simultáneamente, a las condiciones de interpolación que dichas leyes verifican. Esto, como ya se ha mencionado, no se cumple en la misma mediada para la solución clásica de elementos finitos $u^{*}(x)$.

Obsérvese que en el caso del momento puntual, la solución exacta es de la clase $\mathrm{C}^{1}$, en tanto que para la carga concentrada es de la clase $\mathrm{C}^{2}$ y para la carga repartida en parte del vano, es $\mathrm{C}^{3}$. La deformada y las leyes de flectores y cortantes que proporciona el método propuesto son funciones continuas tales que la deformada interpola en el sentido de Hermite, hasta la derivada tercera, en los extremos del elemento, a la solución exacta, los flectores interpolan a los exactos también en el sentido de Hermite hasta la derivada primera, además de ser la diferencia de ambas leyes ortogonal a dos funciones linealmente independientes y, finalmente, el cortante aproximado interpola al exacto en los extremos, solamente en el sentido de Lagrange, pero, a cambio, la diferencia entre ambos es ortogonal a tres funciones linealmente independientes, lo que hace que ambas gráficas se corten en varios puntos intermedios "compensando áreas".

Finalmente, comentamos el caso del momento concentrado, donde los resultados del método propuesto no pueden considerarse óptimos, aunque son claramente mejores que los derivados de la solución por elementos finitos tradicional. En la figura 4(c) se observa la limitada aproximación del momento deducido del método propuesto, al momento exacto, especialmente en las proximidades del salto. Asimismo en la figura 4(d) se pone de manifiesto cómo la ley de cortantes aproximada trata de "recoger", en cierto modo, el cortante exacto incluso en las proximidades del salto del momento donde el cortante exacto viene dado en función de la delta de Dirac.

Obsérvese que aunque no es necesario para la determinación de la deformada y las leyes de esfuerzos, por curiosidad se ha representado la acción repartida equivalente $F(x)$ para cada uno de los casos considerados. Véase apartado (a) de las figuras 2,3 y 4 . Estas cargas repartidas equivalentes son, respectivamente, la proyección ortogonal en el espacio de polinomios de grado menor o igual que tres, de la carga puntual $-12 \delta(x-7) t$, de la carga repartida de $-4 t / m$ en el tramo $x \in[5,5 \mathrm{~m}, 8,5 \mathrm{~m}]$ y nula en el resto del vano, y del momento puntual $-15 \delta^{\prime}(x-7) m x t$. 
CARGA PUNTUAL

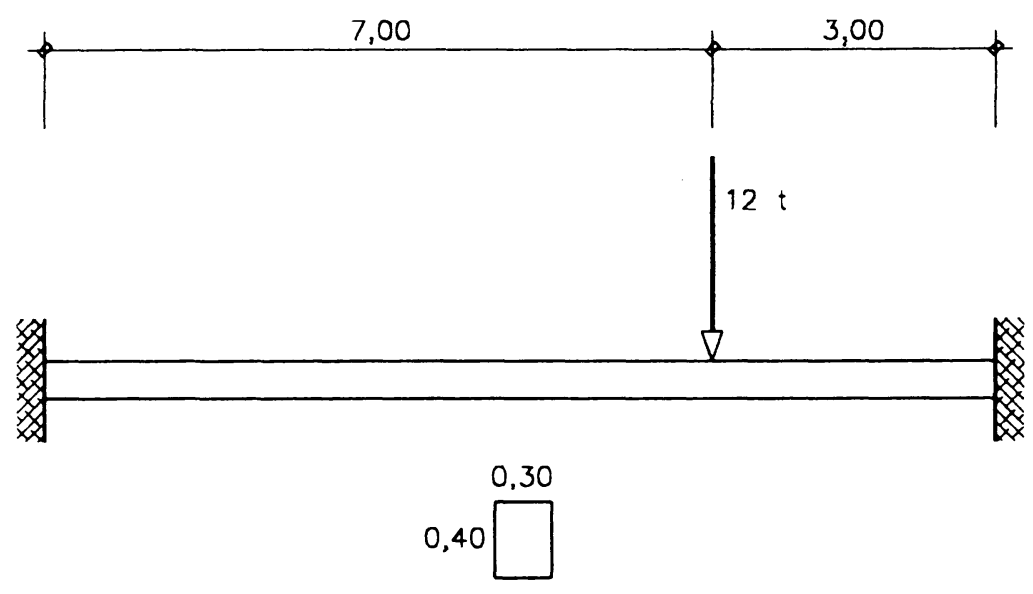

CARGA REPARTIDA EN PARTE DE VANO

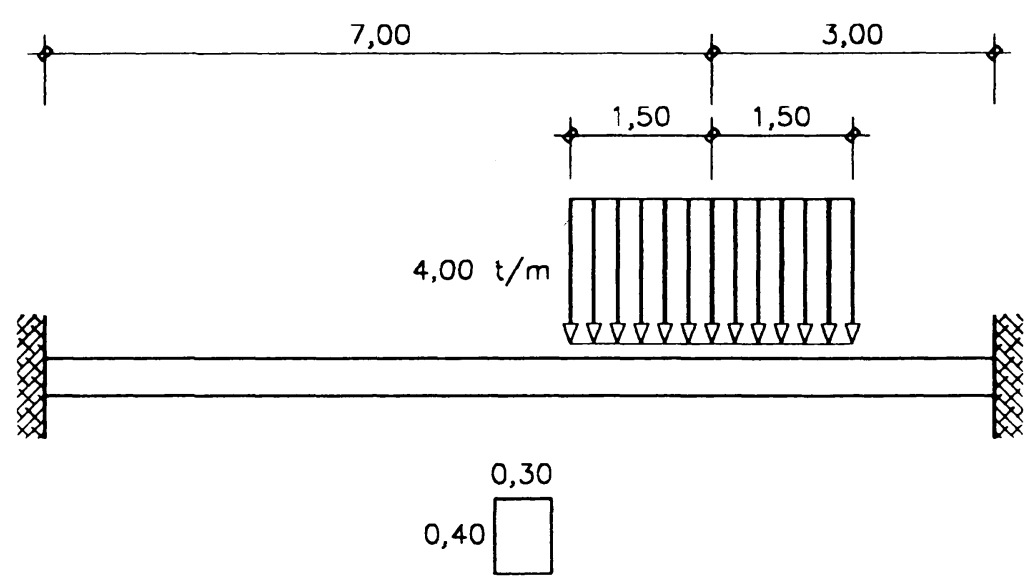

MOMENTO PUNTUAL

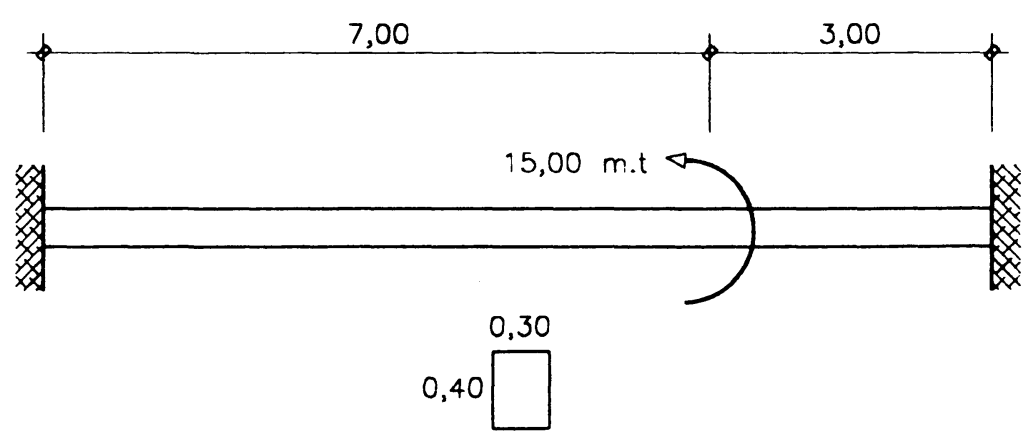

Figura 1 


\section{LEYES PARA LA CARGA PUNTUAL}

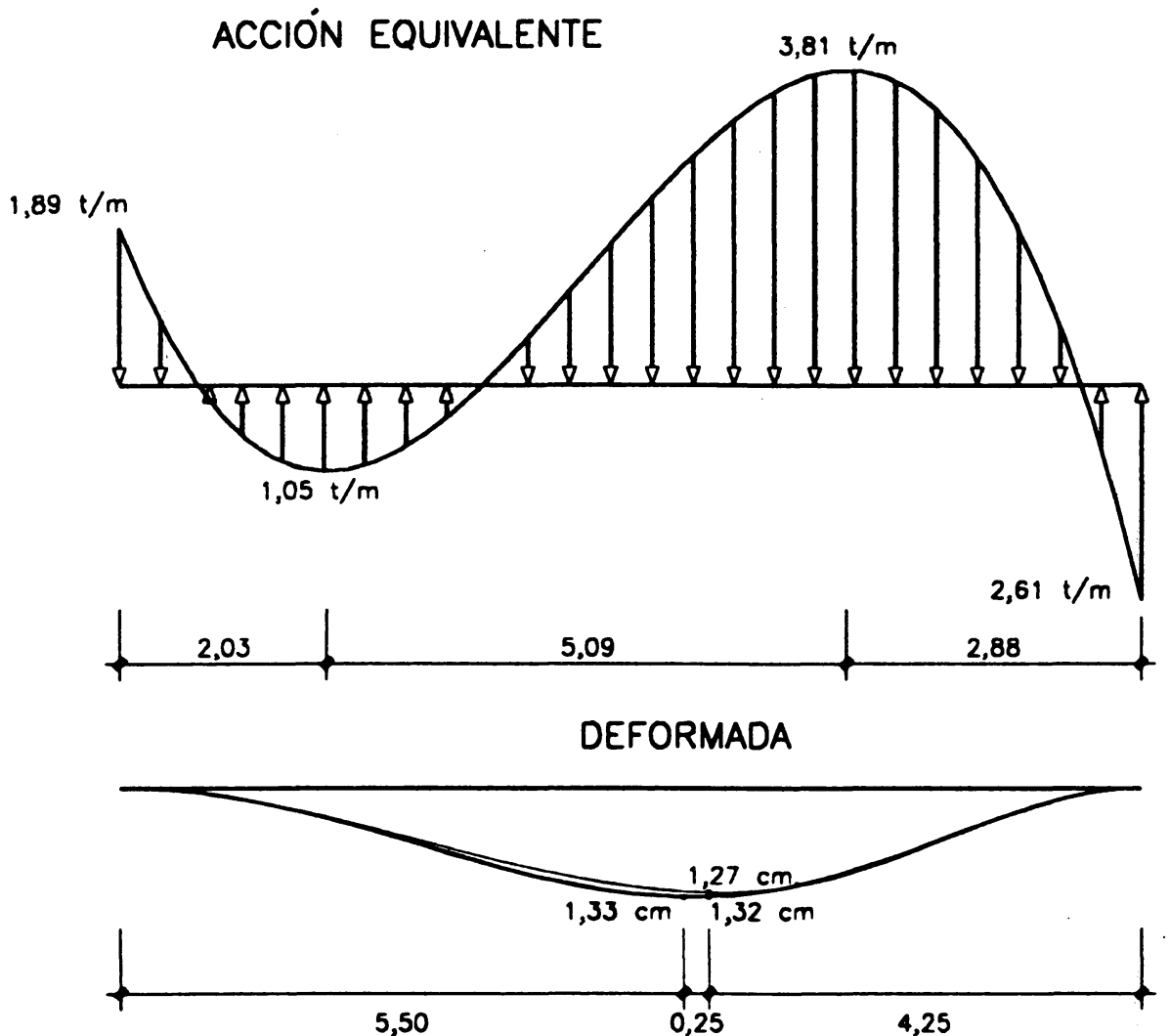

(a)

(b)

(c)

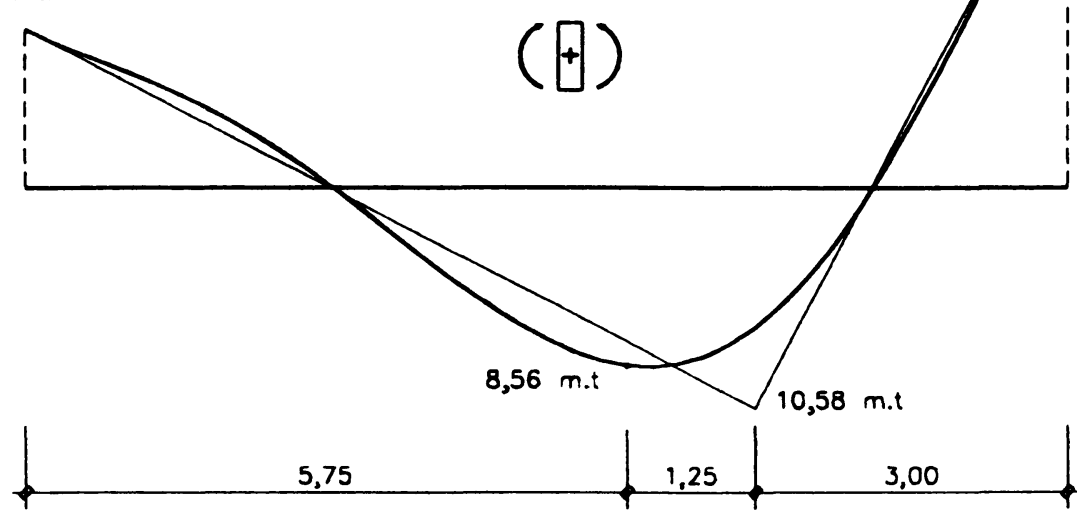

(d)

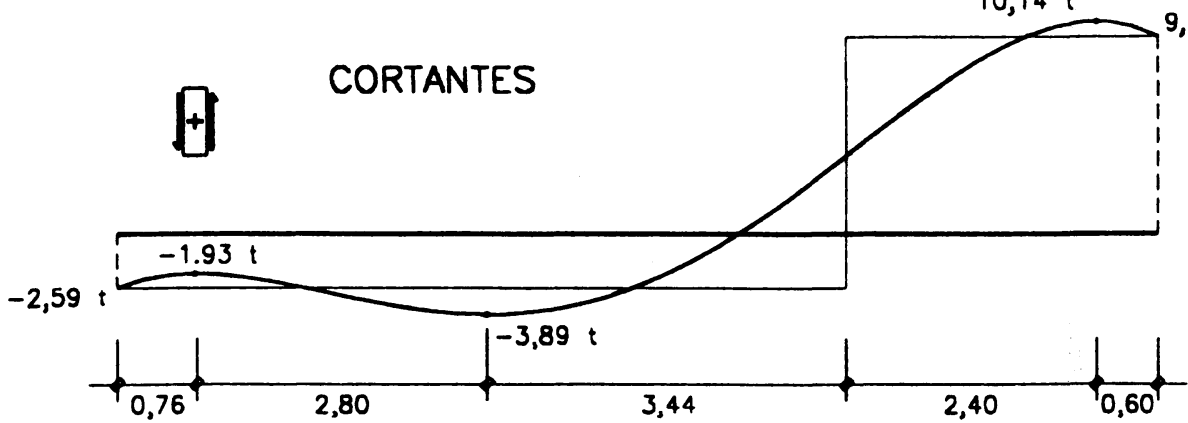

LYES EXACTAS

LEYES MÉTODO PROPUESTO.

Figura 2 
LEYES DE LA CARGA REPARTIDA EN UNA PARTE DEL VANO ACCIÓN EQUIVALENTE

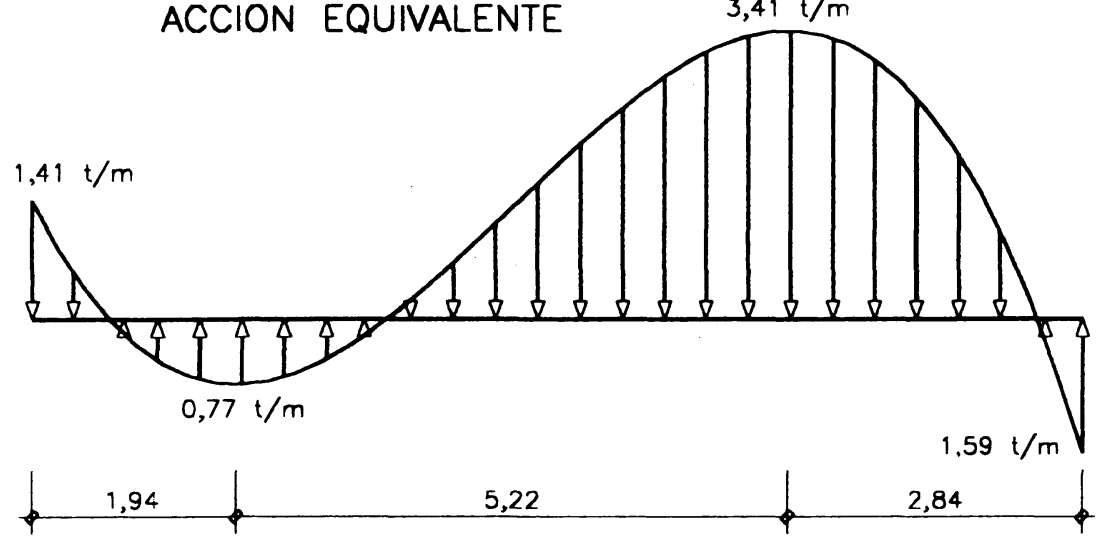

(a)

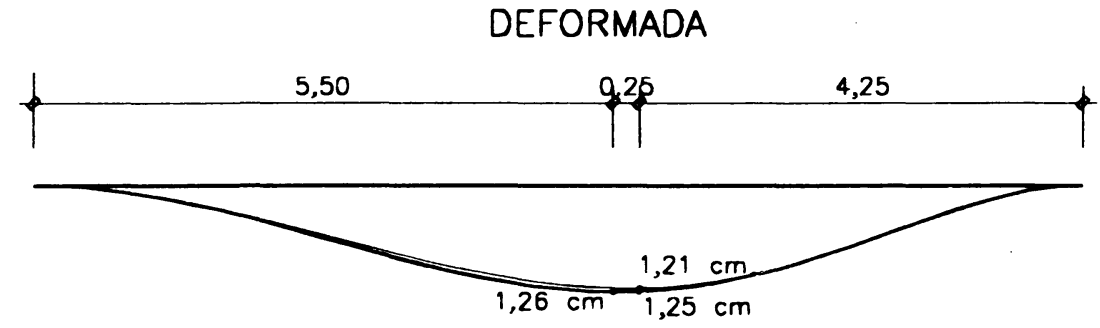

(b)

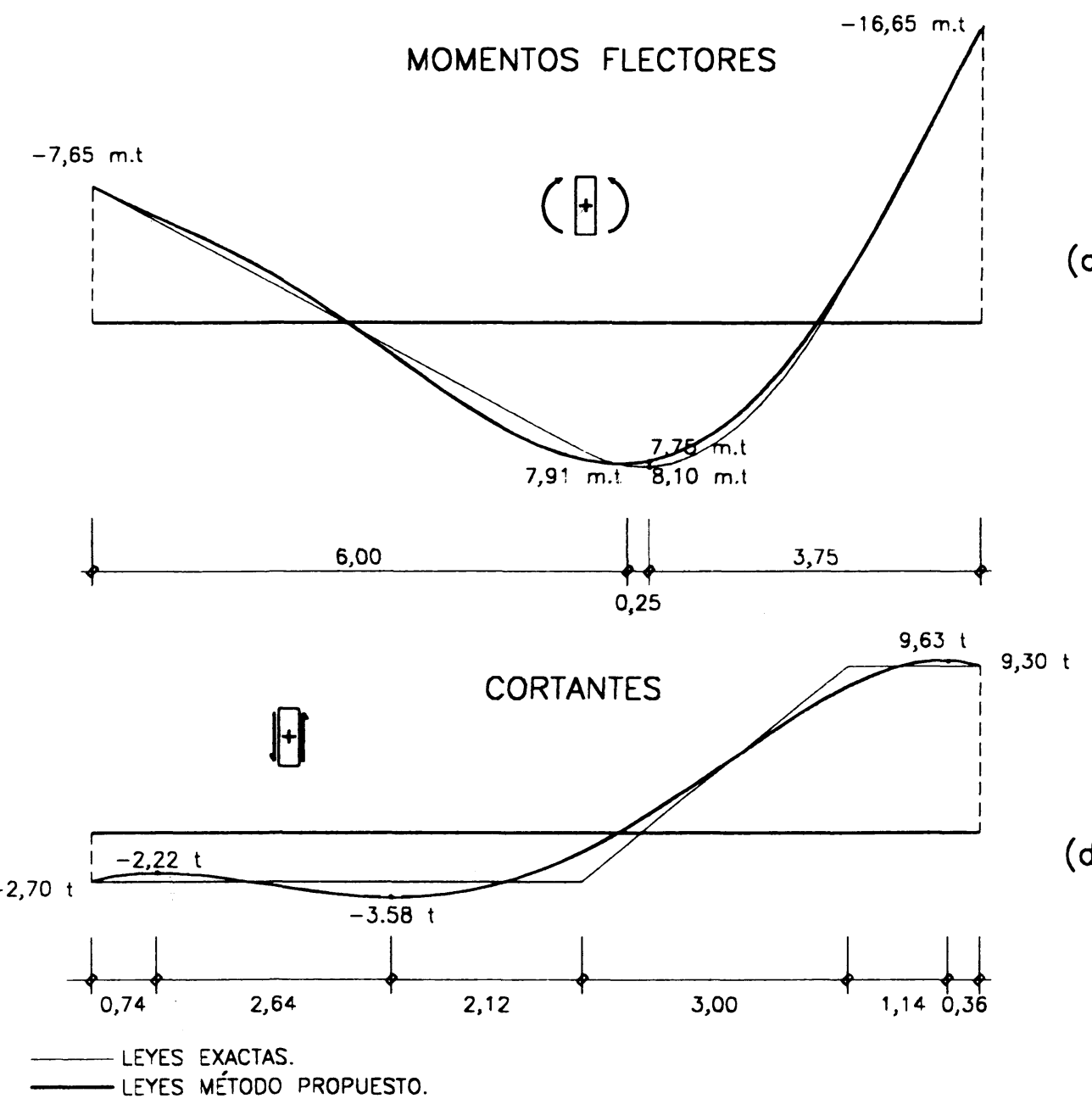

(c)

(d) 
LEYES PARA EL MOMENTO PUNTUAL

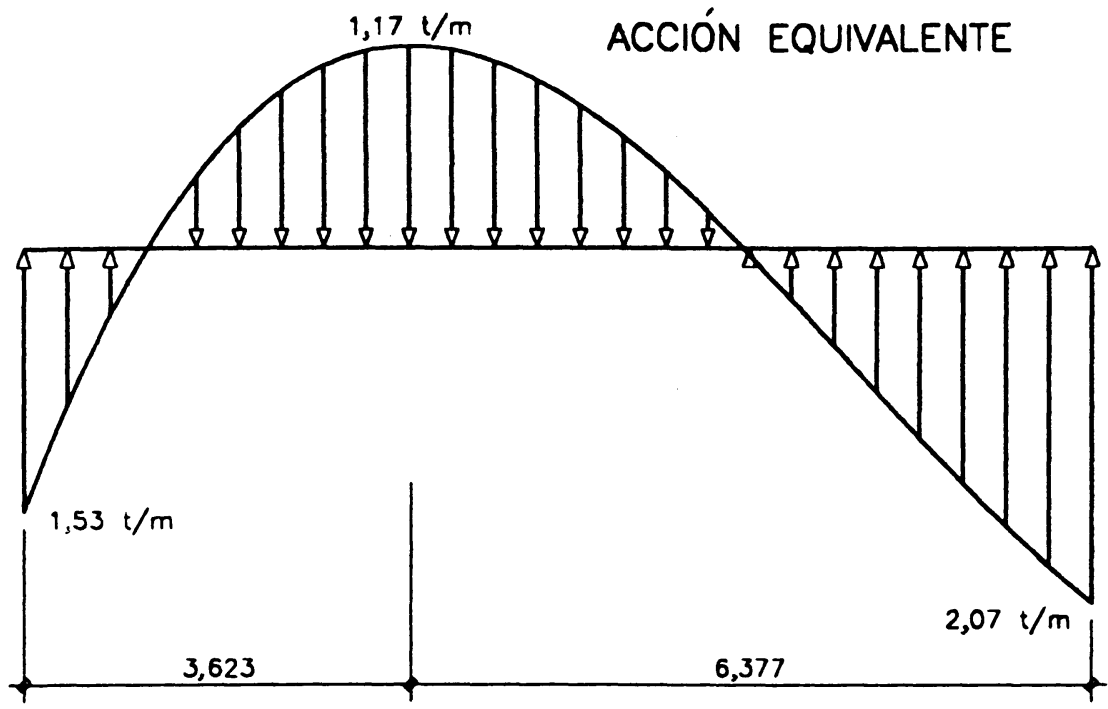

(a)

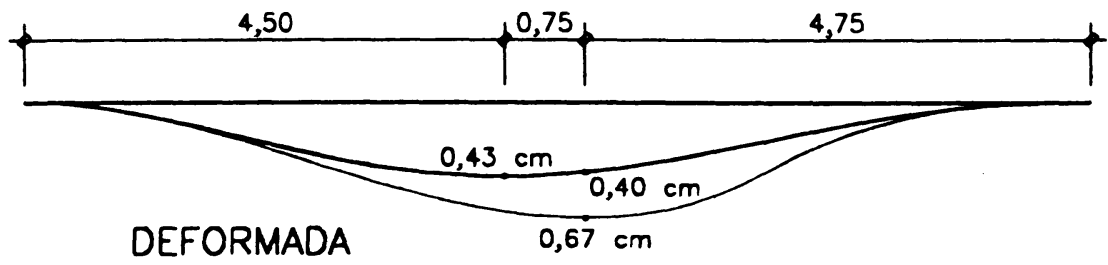

(b)
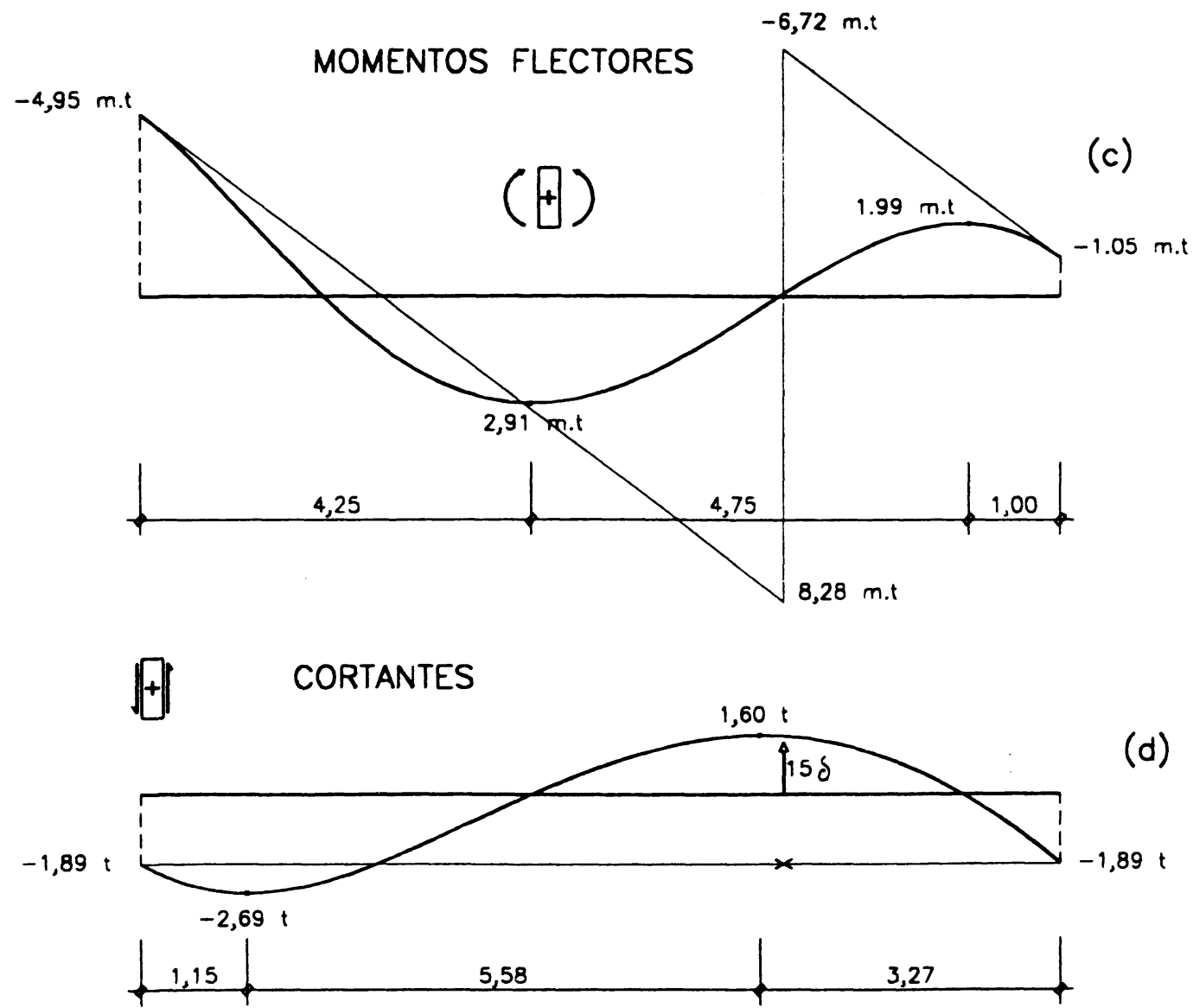

LEYES EXACTAS

LEYES MÉTODO PROPUESTO. 


\subsection{Ejemplo 2}

Este caso, figura 5, corresponde al de una viga biempotrada de $18 \mathrm{~m}$ de longitud con rigidez constante en cada uno de los tres tramos de los que se compone. Se ha considerado solamente un elemento finito por tramo. De esta manera nos hemos situado en el caso más desfavorable en cuanto a tamaño de elementos se refiere. Las acciones están indicadas en la figura. Asimismo las rigideces de cada tramo, para un módulo de elasticidad de $2,1 \times 10^{6} \mathrm{t} / \mathrm{m}^{2}$ y secciones las indicadas en la figura, son, respectivamente, $18.007,5 \mathrm{t} \cdot \mathrm{m}^{2}, 6.562,5 \mathrm{t} \cdot \mathrm{m}^{2}$ y $11.340 \mathrm{t} \cdot \mathrm{m}^{2}$. Los desplazamientos y giros en los nodos 1 , 2,3 y 4 son, respectivamente

$$
\begin{gathered}
u_{1}=u_{1}^{\prime}=0, u_{2}=-0,04296 m, u_{2}^{\prime}=-0,01698, \\
u_{3}=-0,08199 m, u_{3}^{\prime}=0,01572, u_{4}=u_{4}^{\prime}=0
\end{gathered}
$$

donde los dos primeros y los dos últimos son datos y los correspondientes a los nodos intermedios se han obtenido, en la forma usual, de la ecuación de equilibrio global. Dichos valores son, como es conocido, exactos.

Entrando ahora en la ecuación de equilibrio de cada elemento se obtienen las cargas nodales de equilibrio para cada elemento y de dichos valores se obtienen las derivadas segundas y terceras de los desplazamientos en los extremos de cada elemento los cuales resultan, por lo ya indicado, también exactos. Estos valores son, para el primer tramo

$$
\begin{aligned}
& u^{\prime \prime}\left(0^{+}\right)=-0,007630, u^{\prime \prime}\left(0^{+}\right)=0,001734 \\
& u^{\prime \prime}\left(4^{-}\right)=-0,0009047, u^{\prime \prime}\left(4^{-}\right)=0,001545
\end{aligned}
$$

para el segundo tramo de viga

$$
\begin{aligned}
& u^{\prime \prime}\left(4^{+}\right)=-0,002483, u^{\prime \prime}\left(4^{+}\right)=0,003478 \\
& u^{\prime \prime}\left(12^{-}\right)=0,003888, u^{\prime \prime}\left(12^{-}\right)=-0,001886
\end{aligned}
$$

$\mathrm{y}$, finalmente, para el tramo tercero

$$
\begin{aligned}
& u^{\prime \prime}\left(12^{+}\right)=0,002250, u^{\prime \prime}\left(12^{+}\right)=-0,001091 \\
& u^{\prime \prime}\left(18^{-}\right)=-0,009015, u^{\prime \prime}\left(18^{-}\right)=-0,002370
\end{aligned}
$$

Con los ocho valores $u^{(i)}(a), u^{(i)}(b), i=0,1,2,3$ por cada tramo, mediante la expresión (26) se ha determinado la solución aproximada $U(x)$ en cada uno de los tres tramos. A partir de dicha función, mediante derivación y multiplicación por la correspondiente rigidez, se han determinado las leyes de flectores y cortantes aproximadas. Asimismo se han calculado también para cada uno de los tramos, las acciones repartidas equivalentes las cuales se han representado en la figura 6.
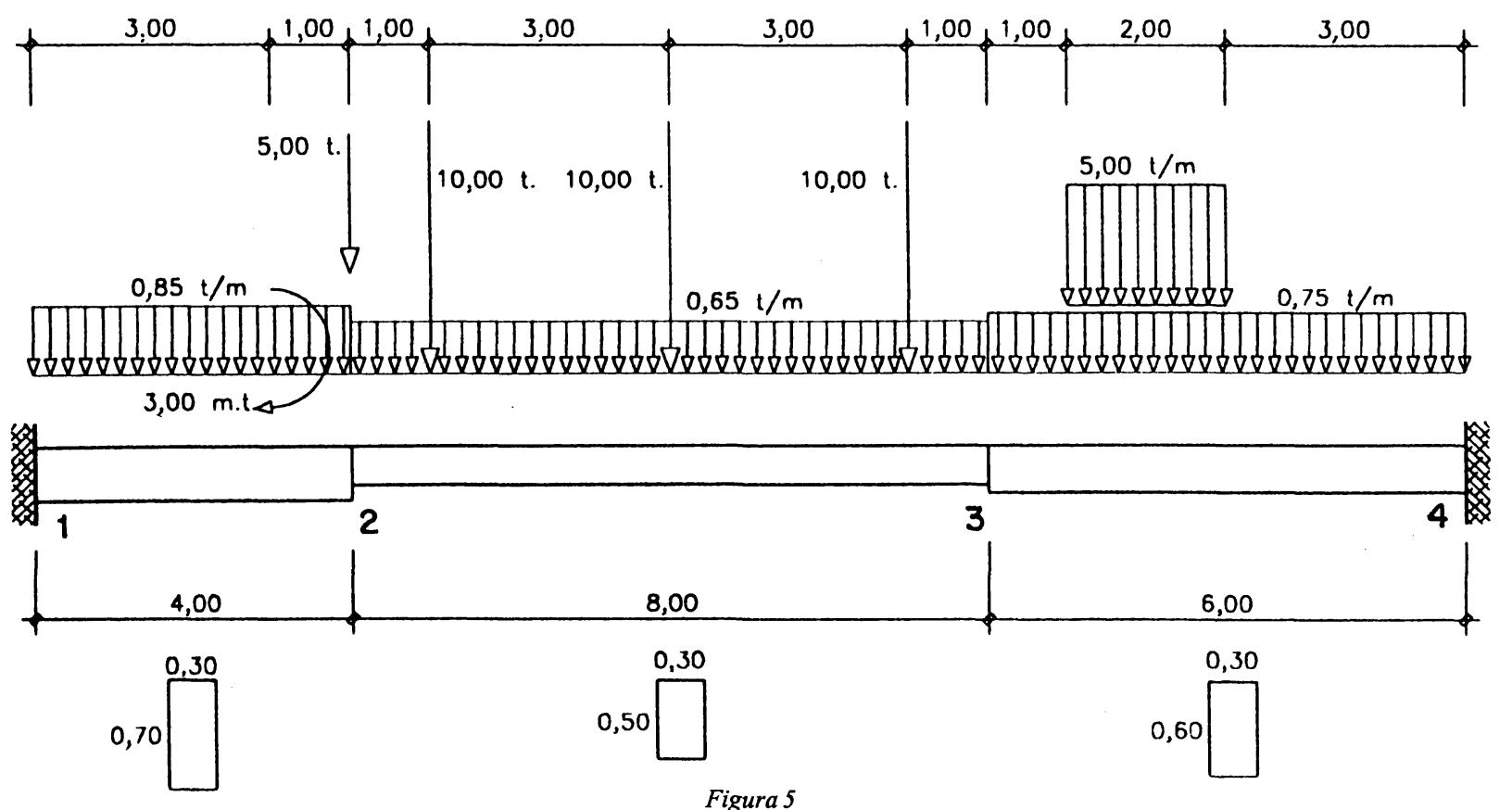

Figura 5 

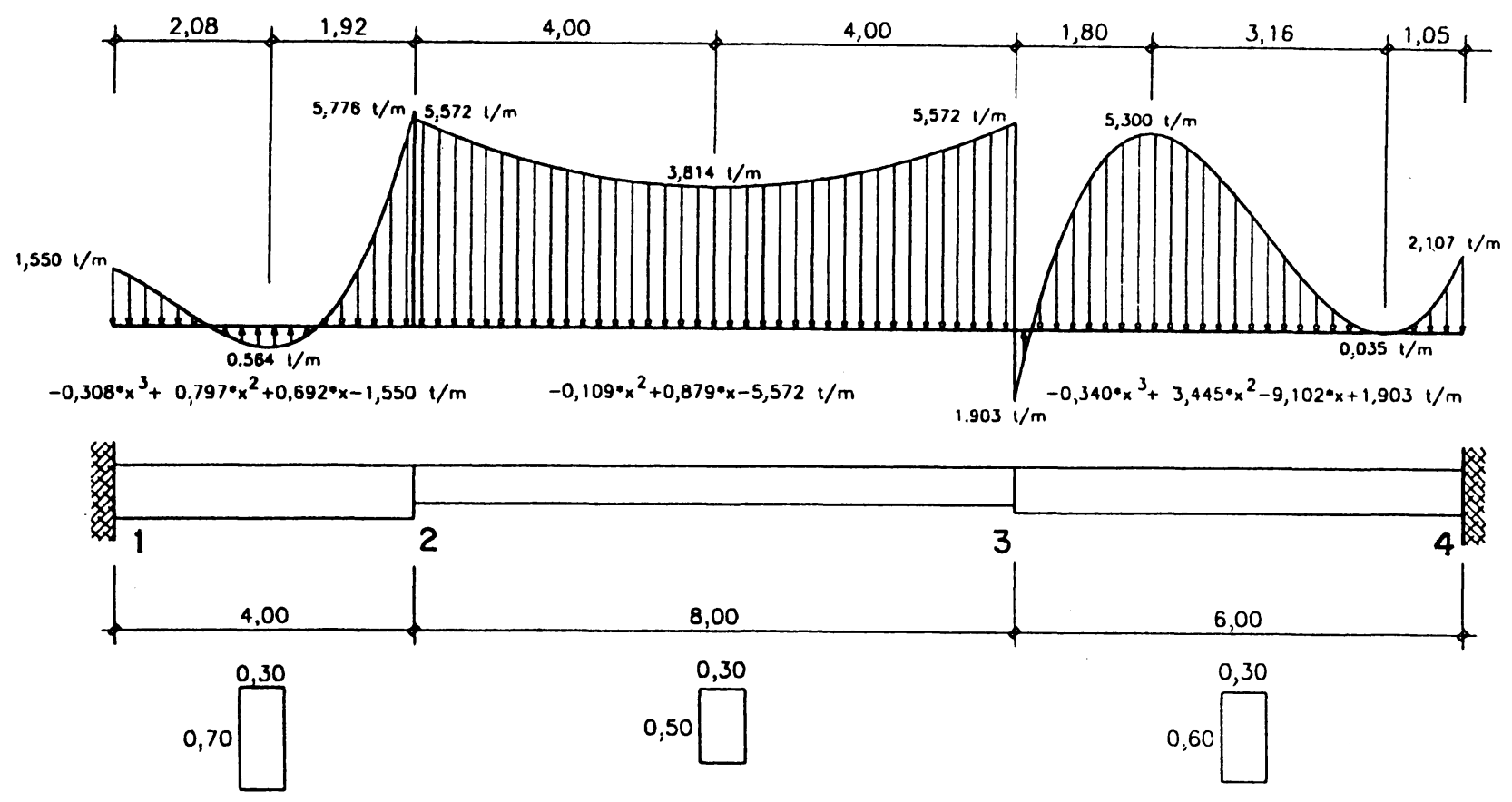

Figura 6

En la tabla 1 se indican los valores numéricos de los desplazamientos evaluados en veintiún puntos igualmente espaciados de cada tramo, donde puede hacerse una comparación de los resultados calculados, por el método propuesto, por el método de elementos finitos y de manera exacta. La gráfica de la deformada se representa en la figura 7 pudiéndose comparar la deformada exacta con la obtenida por el método de elementos finitos y asimismo la deformada calculada por el método propuesto y la obtenida por elementos finitos. Obsérvese que no se han representado simultáneamente la deformada exacta y la calculada por el método propuesto ya que, prácticamente, ambas gráficas se hubieran superpuesto.

TABLA 1

DEFORMADA (CM)

\begin{tabular}{|c|c|c|c|c|c|c|c|c|c|c|c|}
\hline \multicolumn{4}{|c|}{ 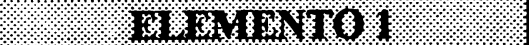 } & \multicolumn{4}{|c|}{ 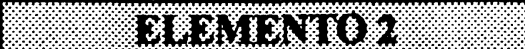 } & \multicolumn{4}{|c|}{$\frac{1}{3}$} \\
\hline $\mathbf{N}^{\circ}$ & EXACTA & MET. P. & E. F. 3 & $\mathbf{N}^{\mathbf{0}}$ & EXACTA & MET. P. & E. F. 3 & $\mathbf{N}^{\circ}$ & EXACTA & MET. P. & E. F. 3 \\
\hline 1 & $-0,000$ & $-0,000$ & $-0,000$ & 21 & $-4,296$ & $-4,296$ & $-4,296$ & 41 & $-8,198$ & $-8,198$ & $-8,198$ \\
\hline 2 & $-0,015$ & $-0,015$ & $-0,015$ & 22 & $-4,991$ & $-4,991$ & $-4,967$ & 42 & -7.717 & $-7,717$ & $-7,713$ \\
\hline 3 & $-0,059$ & $-0,059$ & $-0,059$ & 23 & $-5,704$ & $-5,706$ & $-5,619$ & 43 & $-7,219$ & $-7,219$ & $-7,205$ \\
\hline 4 & $-0,131$ & $-0,131$ & $-0,131$ & 24 & $-6,414$ & -6.420 & $-6,246$ & 44 & $-6,706$ & $-6,706$ & $-6,678$ \\
\hline 5 & $-0,229$ & $-0,229$ & $-0,229$ & 25 & $-7,102$ & $-7,116$ & $-6,843$ & 45 & $-6,182$ & $-6,181$ & $-6,138$ \\
\hline 6 & $-0,352$ & $-0,352$ & $-0,352$ & 26 & $-7,575$ & $-7,779$ & $-7,406$ & 46 & $-5,650$ & $-5,649$ & $-5,591$ \\
\hline 7 & $-0,499$ & $-0,500$ & $-0,500$ & 27 & $-8,369$ & $-8,395$ & $-7,929$ & 47 & $-5,114$ & $-5,113$ & $-5,041$ \\
\hline 8 & $-0,669$ & $-0,669$ & $-0,669$ & 28 & $-8,925$ & $-8,952$ & $-8,406$ & 48 & -4.577 & $-4,576$ & $-4,493$ \\
\hline 9 & $-0,859$ & $-0,860$ & $-0,860$ & 29 & $-9,416$ & $-9,441$ & $-8,834$ & 49 & $-4,045$ & $-4,044$ & $-3,954$ \\
\hline 10 & $-1,069$ & $-1,070$ & $-1,070$ & 30 & $-9,830$ & $-9,851$ & $-9,206$ & 50 & $-3,521$ & $-3,521$ & $-3,428$ \\
\hline 11 & $-1,297$ & $-1,298$ & $-1,298$ & 31 & $-10,157$ & $-10,176$ & $-9,518$ & 51 & $-3,012$ & $-3,012$ & $-2,920$ \\
\hline 12 & $-1,543$ & $-1,544$ & $-1,544$ & 32 & $-10,388$ & $-10,409$ & $-9,764$ & 52 & $-2,522$ & $-2,524$ & $-2,436$ \\
\hline 13 & $-1,804$ & $-1,805$ & $-1,805$ & 33 & $-10,522$ & $-10,547$ & $-9,940$ & 53 & $-2,059$ & $-2,060$ & $-1,980$ \\
\hline 14 & $-2,079$ & $-2,081$ & $-2,081$ & 34 & $-10,560$ & $-10,587$ & $-10,041$ & 54 & $-1,626$ & $-1,628$ & $-1,558$ \\
\hline 15 & $-2,368$ & $-2,369$ & $-2,369$ & 35 & $-10,501$ & $-10,527$ & $-10,060$ & 55 & $-1,232$ & $-1,233$ & $-1,176$ \\
\hline 16 & $-2,669$ & $-2,669$ & $-2,669$ & 36 & $-10,345$ & $-10,367$ & $-9,994$ & 56 & $-0,881$ & $-0,882$ & $-0,838$ \\
\hline 17 & $-2,979$ & $-2,980$ & $-2,979$ & 37 & $-10,095$ & $-10,110$ & -9.837 & 57 & $-0,580$ & $-0,581$ & $-0,550$ \\
\hline 18 & $-3,299$ & $-3,299$ & $-3,299$ & 38 & $-9,751$ & $-9,758$ & $-9,584$ & 58 & $-0,335$ & $-0,336$ & $-0,317$ \\
\hline 19 & $-3,625$ & $-3,625$ & $-3,625$ & 39 & $-9,315$ & $-9,317$ & -9.230 & 59 & $-0,153$ & $-0,153$ & $-0,144$ \\
\hline 20 & $-3,958$ & $-3,958$ & $-3,958$ & 40 & $-8,794$ & $-8,794$ & $-8,770$ & 60 & $-0,038$ & $-0,039$ & $-0,037$ \\
\hline 21 & $-4,296$ & $-4,296$ & $-4,296$ & 41 & $-8,198$ & $-8,198$ & $-8,198$ & 61 & 0,000 & 0,000 & 0,000 \\
\hline
\end{tabular}




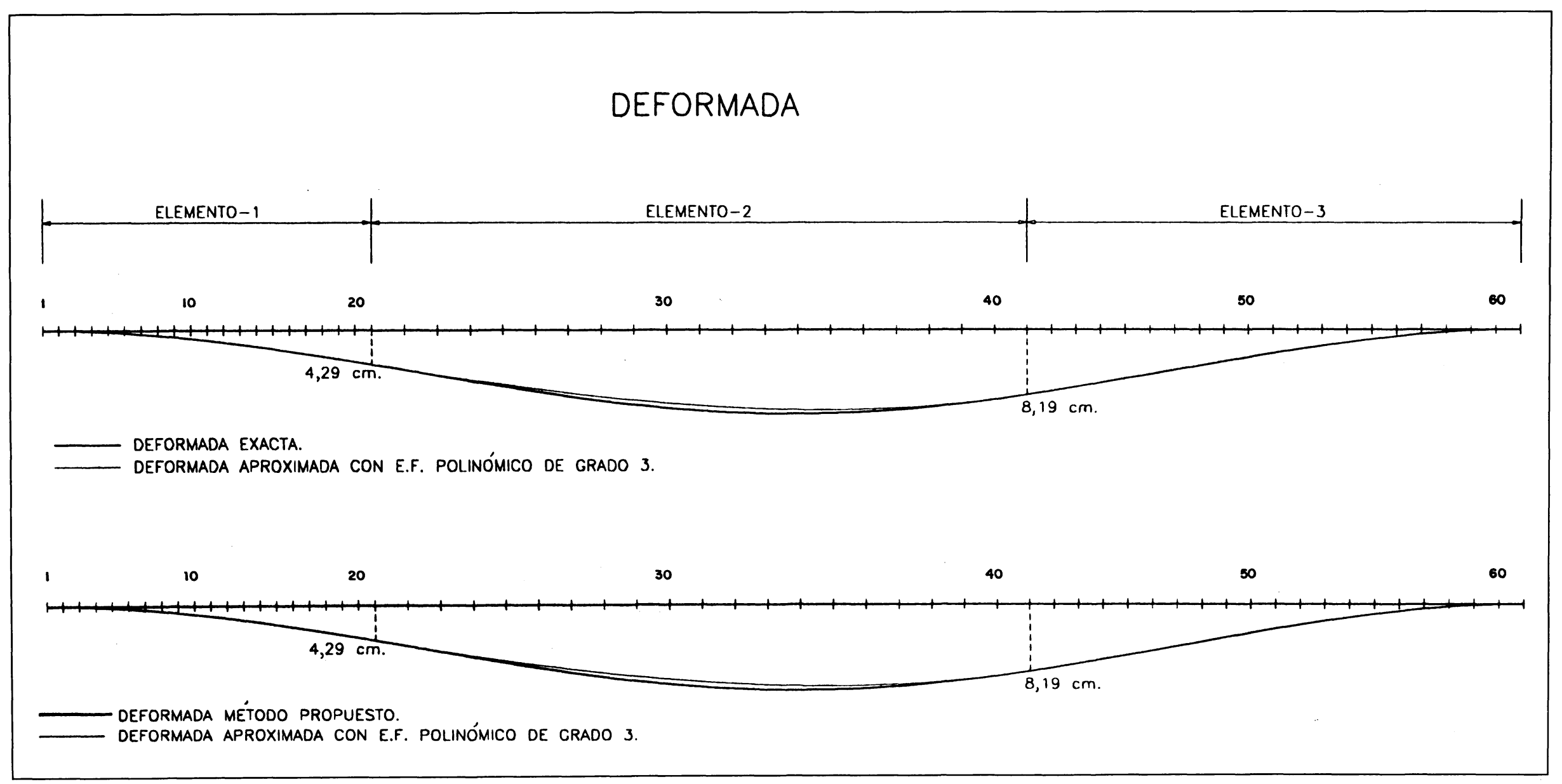

Figura 7 
En la tabla 2 se dan los valores del momento flector exactos y los obtenidos por el método propuesto y en la figura 8 se representan las gráficas correspondientes, incluyendo los momentos flectores que se deducen de la solución obtenida por elementos finitos. Esta última gráfica representada con línea de trazos se aleja, como puede verse, de manera considerable de la ley exacta de flectores y de la calculada por el método propuesto, las cuales son a su vez muy próximas entre sí. Destacamos aquí que la ley de flectores que da el método propuesto, es continua globalmente en este caso, debido a que no hay ningún momento exterior aplicado en los dos nodos de separación de los tramos. Asimismo puede observarse que, dentro de cada elemento, también la ley es continua, aunque no lo sea la ley exacta de flectores, pues el método, en definitiva, lo que hace es regularizar la acción dentro del elemento. Nótese que las leyes que proporciona el método son leyes exactas para la acción repartida equivalente.

TABLA 2

MOMENTO (M. T.)

\begin{tabular}{|c|c|c|c|c|c|c|c|c|}
\hline \multicolumn{3}{|c|}{ 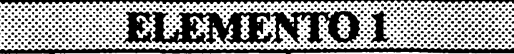 } & \multicolumn{3}{|c|}{ 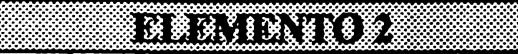 } & \multicolumn{3}{|c|}{ 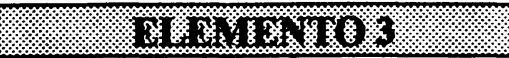 } \\
\hline $\mathbf{N}^{\circ}$ & EXACTO & MET. P. & $\mathbf{N}^{\mathbf{0}}$ & EXACTO & MET. P. & $\mathbf{N}^{\circ}$ & EXACTO & MET. P. \\
\hline 1 & $-137,39$ & $-137,39$ & 21 & -16.29 & $-16,29$ & 41 & 25,51 & 25.51 \\
\hline 2 & $-131,16$ & $-131,18$ & 22 & $-7,21$ & $-7,59$ & 42 & 21,77 & 21,85 \\
\hline 3 & $-124,97$ & $-125,02$ & 23 & 1.76 & 0,25 & 43 & 17,96 & 18,14 \\
\hline 4 & $-118,81$ & $-118,90$ & 24 & 8.63 & 7,32 & 44 & 14,08 & 14,22 \\
\hline 5 & $-112,68$ & $-112,83$ & 25 & 13,40 & 13,63 & 45 & 10,03 & 9,97 \\
\hline 6 & $-106,59$ & $-106,77$ & 26 & 18,06 & 19,24 & 46 & 5,49 & 5,30 \\
\hline 7 & $-100,53$ & $-100,74$ & 27 & 22.62 & 24.16 & 47 & 0,43 & 0,17 \\
\hline 8 & $-94,51$ & -94.70 & 28 & 27.07 & 28,43 & 48 & $-5,14$ & -5.43 \\
\hline 9 & $-88,52$ & -82.62 & 29 & 31,42 & 32.06 & 49 & $-11,23$ & $-11,49$ \\
\hline 10 & $-82,56$ & -82.62 & 30 & 35,67 & 35,07 & 50 & $-17,84$ & $-17,99$ \\
\hline 11 & $-76,64$ & $-76,55$ & 31 & 39,81 & 37,46 & 51 & $-24,97$ & $-24,87$ \\
\hline 12 & $-70,75$ & $-70,45$ & 32 & 39.85 & 39,25 & 52 & $-32,39$ & -32.07 \\
\hline 13 & $-64,90$ & $-64,34$ & 33 & 39.78 & 40,42 & 53 & $-39,88$ & -39.52 \\
\hline 14 & $-59,08$ & $-58,21$ & 34 & 39.61 & 40,97 & 54 & $-47,43$ & -47.17 \\
\hline 15 & $-53,29$ & $-52,07$ & 35 & 39.34 & 40,88 & 55 & -55.06 & $-54,93$ \\
\hline 16 & $-44,54$ & $-45,94$ & 36 & 38.96 & 40,14 & 56 & $-62,75$ & -62.76 \\
\hline 17 & $-38,82$ & $-39,83$ & 37 & 38,48 & 38,72 & 57 & $-70,51$ & $-70,62$ \\
\hline 18 & $-33,14$ & -33.77 & 38 & 37,89 & 36,58 & 58 & $-78,33$ & $-78,48$ \\
\hline 19 & $-27,49$ & -27.80 & 39 & 35.20 & 33,70 & 59 & $-86,23$ & -86.34 \\
\hline 20 & $-21,87$ & $-21,96$ & 40 & 30.41 & 30.02 & 60 & $-94,19$ & -94.23 \\
\hline 21 & $-16,29$ & $-16,29$ & 41 & 25.51 & 25,51 & 61 & $-102,22$ & $-102,22$ \\
\hline
\end{tabular}




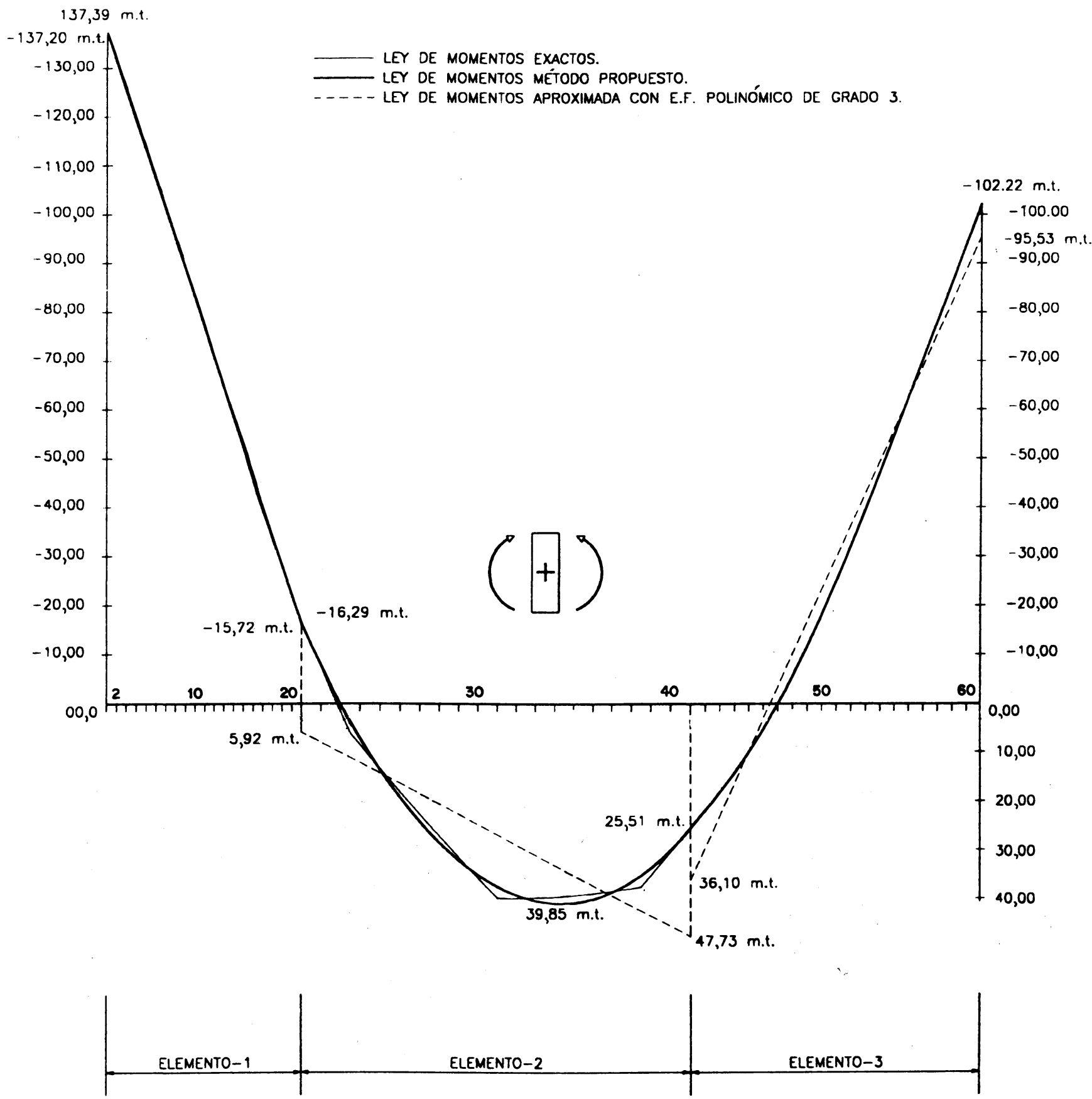

Figura 8

A continuación, en la tabla 3, se indican, para los veintiún puntos en los que se ha dividido cada tramo de viga, los valores del esfuerzo cortante, representándose en la figura 9 las gráficas de dicha ley para los métodos considerados. De nuevo puede observarse, aquí también, el efecto regularizante del método propuesto. Aunque en este caso la ley de cortantes que da el método es discontinua debido a la existencia de una carga puntual en el nodo de separación de los dos primeros tramos. De nuevo se pone de manifiesto la propiedad, del cortante calculado por el método propuesto, de aproximar al cortante exacto incluso en las zonas en las que éste viene dado en función de la delta de Dirac. Esto último ocurre en el primer tramo de viga, en el punto de aplicación del momento puntual de $-3 \mathrm{~m} \mathrm{x} \mathrm{t}$ donde el cortante exacto viene dado por $-3 \delta$, y el cortante que proporciona el método trata de "recoger" dicho valor. Precisamente, si no se repara en lo anterior, podría pensarse, que en un entorno del punto indicado, la ley de cortantes del método propuesto, se aleja de la ley de cortantes exacta, tal y como aparentemente sugieren las gráficas de la figura 9 en el primer tramo de viga. 
TABLA 3

CORTANTE (T)

\begin{tabular}{|c|c|c|c|c|c|c|c|c|}
\hline \multicolumn{3}{|c|}{ W } & \multicolumn{3}{|c|}{ 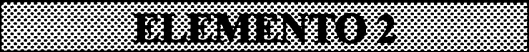 } & \multicolumn{3}{|c|}{ 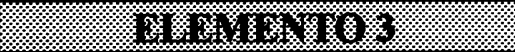 } \\
\hline $\mathbf{N}^{0}$ & EXACTO & MET. P. & $\mathrm{N}^{\circ}$ & EXACTO & MET. P. & $\mathbf{N}^{0}$ & EXACTO & MET. P. \\
\hline 1 & $-31,22$ & $-31,22$ & 21 & $-27,82$ & $-27,82$ & 41 & 12,37 & 12,37 \\
\hline 2 & $-31,05$ & $-30,93$ & 22 & $-22,56$ & $-20,66$ & 42 & 12,59 & 12,58 \\
\hline 3 & \begin{tabular}{|c|}
$-30,88$ \\
\end{tabular} & $-30,67$ & 23 & $-22,30$ & $-18,63$ & 43 & 12,82 & 12,63 \\
\hline 4 & $-30,71$ & $-30,46$ & 24 & $-12,04$ & $-16,70$ & 44 & 13,04 & 13,56 \\
\hline 5 & $-30,54$ & $-30,31$ & 25 & $-11,78$ & $-14,88$ & 45 & 14,27 & 14,83 \\
\hline 6 & $-30,37$ & $-30,21$ & 26 & $-11,52$ & $-13,14$ & 46 & 15,99 & 16,31 \\
\hline 7 & $-30,20$ & $-30,16$ & 27 & $-11,26$ & $-11,47$ & 47 & 17,72 & 17,88 \\
\hline 8 & $-30,03$ & $-30,16$ & 28 & $-11,00$ & $-9,86$ & 48 & 19,44 & 19,46 \\
\hline 9 & $-29,86$ & $-30,21$ & 29 & $-10,74$ & $-8,29$ & 49 & 21,17 & 20,96 \\
\hline 10 & $-29,69$ & $-30,29$ & 30 & $-10,48$ & $-6,75$ & 50 & 22,89 & 22,32 \\
\hline 11 & $-29,52$ & $-30,40$ & 31 & $-0,22$ & $-5,22$ & 51 & 24,62 & 23,50 \\
\hline 12 & $-29,35$ & $-30,51$ & 32 & 0,03 & $-3,69$ & 52 & 24,84 & 24,46 \\
\hline 13 & $-29,18$ & $-30,61$ & 33 & 0,29 & $-2,15$ & 53 & 25,07 & 25,19 \\
\hline 14 & $-29,01$ & $-30,68$ & 34 & 0,55 & $-0,58$ & 54 & 25,29 & 25,71 \\
\hline 15 & $-28,84$ & $-30,69$ & 35 & 0,81 & 1,02 & 55 & 25,52 & 26,02 \\
\hline 16 & $\left({ }^{*}\right)-3 \quad \delta$ & $-30,62$ & 36 & 1,07 & 2,69 & 56 & 25,74 & 26,16 \\
\hline 17 & $-28,50$ & $-30,43$ & 37 & 1,33 & 4,43 & 57 & 25,97 & 26,20 \\
\hline 18 & $-28,33$ & $-30,09$ & 38 & 1,59 & 6,25 & 58 & 26,19 & 26,19 \\
\hline 19 & $-28,16$ & $-29,57$ & 39 & 1185 & 8,17 & 59 & 26,42 & 26,23 \\
\hline 20 & $-27,99$ & $-28,83$ & 40 & 12,11 & 10,21 & 60 & 26,64 & 26,41 \\
\hline 21 & $-27,82$ & $-27,82$ & 41 & 12,37 & 12,37 & 61 & 26,87 & 26,87 \\
\hline
\end{tabular}
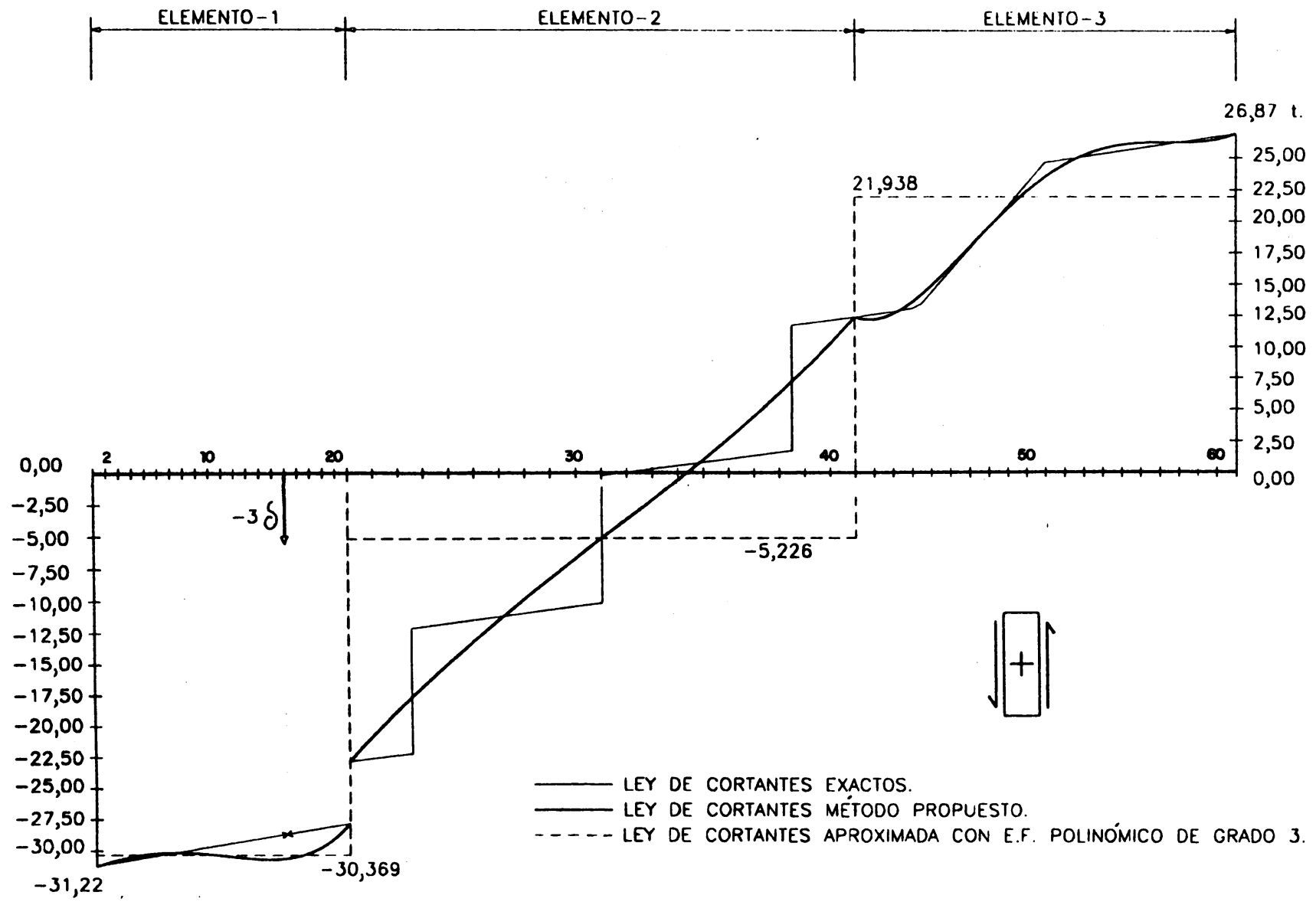
Finalmente, se destaca que para obtener con los elementos finitos hermíticos usuales, resultados semejantes, para la deformada, flectores y cortantes, a los obtenidos mediante la aplicación del método propuesto, se requiere para el caso tratado, discretizar cada tramo de viga en, al menos, diez elementos de igual tamaño. La afirmación anterior viene dada fundamentalmente por la aproximación del cortante, pues es la ley que peor se aproxima y, en consecuencia, la que más condiciona el grado de discretización. Obsérvese que todos los cálculos derivados de la aplicación del método propuesto, en el ejemplo, han sido obtenidos partiendo de un único elemento finito por tramo de viga, lo que ha reducido aproximadamente en un orden de magnitud, el tamaño del sistema de ecuaciones (13).

\section{Conclusiones y comentarios}

Como resumen de lo expuesto puede indicarse que se ha concretado un método que permite optimizar los resultados obtenidos a partir de los elementos finitos hermíticos usuales. La principal ventaja del método es mejorar el grado de precisión de los desplazamientos y esfuerzos en el interior de los elementos, incluso utilizando elementos de gran tamaño. Los valores aproximado que da el método propuesto, tanto para la deformada como para las leyes de esfuerzos, pueden ser interpretados como exactos correspondientes a una nueva acción, repartida, relacionada con el estado de carga original. Dicha acción la hemos denominado acción repartida equivalente y es un representante canónico de una infinidad de estados de carga tales que todos ellos producirían los mismos movimientos y esfuerzos en los extremos de cada elemento.

Se destacan también las propiedades deducidas sobre ortogonalidad e interpolación de las leyes de esfuerzos aproximadas que proporciona el método, así como la comparación con las leyes derivadas de la utilización de los elementos finitos hermíticos usuales. En dicha comparación han sido puestas de manifiesto las interesantes ventajas del método propuesto. Precisamente, además de las propiedades de interpolación que constituyen inicialmente la base del método, son las propiedades de ortogonalidad entre la diferencia de los esfuerzos exactos y los aproximados por el método, con un conjunto de funciones linealmente independientes (que se traduce en una compensación de áreas de las leyes aproximadas con las exactas), lo que hace que el método proporcione unos resultados notablemente aproximados a los exactos en el interior de cada elemento.

Finalmente indicamos que el método, desde el punto de vista práctico, puede extenderse también a problemas de vigas de rigidez constante sobre fundación elástica y a problemas de pandeo de pilares. En estos casos la determinación de la solución de la ecuación homogénea $L^{2} U=0$ que interpola los esfuerzos y desplazamientos puede facilitarse con el empleo de herramientas de cálculo simbólico.

Para los casos de rigidez variable el procedimiento propuesto puede adaptarse fácilmente empleando las mismas funciones polinómicas utilizadas para el caso de rigidez constante. En dicha situación los movimientos y esfuerzos en los nodos no serán exactos, pues son los que resultan directamente de la aplicación del método de los elementos finitos, sin embargo, la aproximación que proporciona el método para desplazamientos y esfuerzos, dentro de cada elemento, es superior a la que se obtiene directamente de la solución por elementos finitos.

\section{BIBLIOGRAFÍA}

[1] TONG, P.: "Exact Solution of Certain Problems by Finite-Element Method", AIAA Journal, Vol. 7, No.1, 1969.

[2] FILHO, F. V.: "Comments on Computation of Stress Resultants from the Element Stiffness Matrices", AIAA Journal, Vol. 6, No. $3,1968$.

[3] STRICKLIN, J. A.: “ Computation of Stress Resultants from the Element Stiffness Matrices”, AIAA Journal, Vol. 4, No. 6, 1966.

[4] BARLOW, J.: "Optimal Stress Locations in Finite Element Method", International Journal for Numerical Methods in Engineering, $10,1976$.

[5] BREBBIA, C. A.: "The Boundary Element Method for Engineers", Pentech Press, London, 1978.

[6] HERRERA, I.: "Theory of connectivity: a systematic formulation of boundary element methods. In Recent Advances in Boundary Element Methods (ed. C. A. Brebbia)", Pentech Press, 1978.

[7] ROMERO, J. L. y ORTEGA, M. A.: "Splines Generalizados y Solución Nodal Exacta en el Método de Elementos Finitos". A publicarse en Informes de la Construcción.

[8] FUNARO, D.: "Polynomial Approximation of Differential Equations", Lectures Notes in Physics, m8, Springer-Verlag, 1992. 
[9] INCE, E. L.: “Ordinary differential equations”, Dover Publ. Inc., New York, 1956.

[10] CODDINGTON E. A. y LEVISON, N.: "Theory of Ordinary Differential Equations”, Mc Graw-Hill, New York, 1955.

[11] STAKGOLD, I.: "Boundary value problems in Mathematical Physics", Vol. I, Mac Millan Co. New York, 1968.

[12] SCHWARTZ, L.: “Métodos Matemáticos para las Ciencias Fisicas”, Selecciones Científicas, Madrid, 1969.

[13] DAVIS, P. J. y RABINOWITZ, P., "Methods of Numerical Integration”, Academic Press, Inc. 1984

[14] DAVIS, P. J.,"Interpolation and Approximation", Dover Publ. Inc. New York, 1975.

\section{publicaciones del IETCC/CSIC}

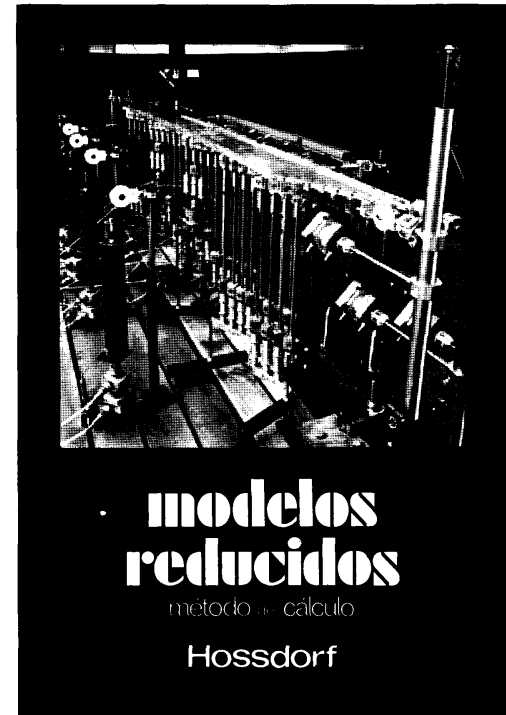

Modelos reducidos. Método de cálculo

H. Hossdorf, Ingeniero Civil

La técnica de los ensayos en modelos reducidos de estructuras sufre hoy dia una decisiva metamorfosis. Hasta hace poco era un medio más bien de artesania, que no siempre era tomado en serio por los académicos teorizantes Dara comprender el comportamiento resistente de las estructuras complejas $y$ a que se acudió las más de las veces, como a un ultimo remedio debido a sus indiscutibles insuficien cias. Sin embargo, en poco tiempo y gracias a su conexión con los ordenadores digitales, se ha transformado en un instrumento cientificamente valioso que no puede quedar a un lado en la práctica diaria del Ingeniero Proyectista.

Un volumen encuadernado en cartoné plastificado con lomo de tela de $17 \times 24 \mathrm{~cm}$. compuesto de 250 páginas, 158 figuras $y$ fotografias.

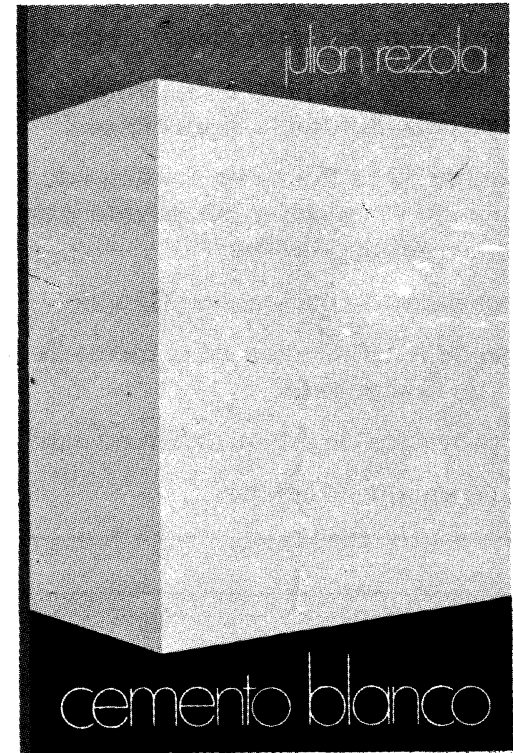

\section{Cemento blanco}

Julián Rezola

Ingeniero Químico Dipl. I. Q. S

Sabido es que existe una extensa y documentada bibliografia sobre el cemento gris: en cambio, no puede decirse lo mismo acerca del cemento portland blanco, ya que los escritos existentes se refieren tan sólo a algunas peculiaridades que le distinguen de aquél.

El autor nos ofrece sus profundos conocimientos $y$ su larga experiencia tanto en laboratorio como y su larga experi

La parte descriptiva del libro se complementa con gráficos, diagramas y fotografias de gran utilidad destinados a conseguir la aplicación apropiada de este aglomerante.

Un volumen encuadernado en cartoné policerado, de $17,4 \times 24,3 \mathrm{~cm}$, compuesto de 395 páginas

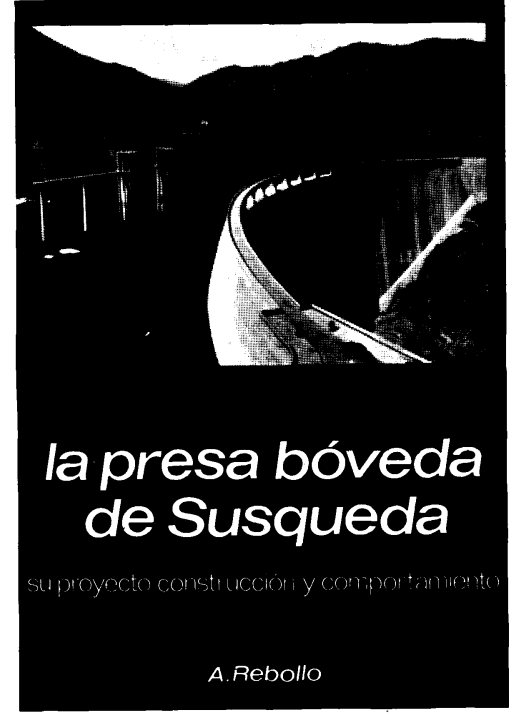

La presa bóveda de Susqueda

A. Rebollo,

Dr. Ingeniero de Caminos

El esfuerzo del constructor de presas se sitúa, por su pretensión de perennidad, a contracorriente de las tendencias de la civilización actual, caracterizada por lo fungible. Pueden evocarse las 10.000 grandes. presas en funcionamiento o en construcción que están envejeciendo y reclaman los cuidados gerontológicos para mantener y perfeccionar su servicio y garantizar su inalienable pretensión de perennidad. En la medida en que todas nuevas obras, grandes o pequeñas, son portadoras de riesgos ecológicos $y$, a veces, catastróficos, que aumentan con el envejecimiento, la gerontologia de las presas es todo un emplazo. La accion adelantada de Arturo Rebollo en este terreno marca un camino a seguir para todos los que aman su propia obra con la devoción paternal que él ha puesto en Susqueda.

Un volumen encuadernado en cartoné plastificado con lomo de tela, de $18 \times 24,5 \mathrm{~cm}$, compuesto de 408 páginas, 330 figuras $y$ fotografias y 39 tablas. 


\title{
Publicaciones del Instituto Eduardo Torroja - CSIC
}

\author{
Números monográficos de INFORMES
}
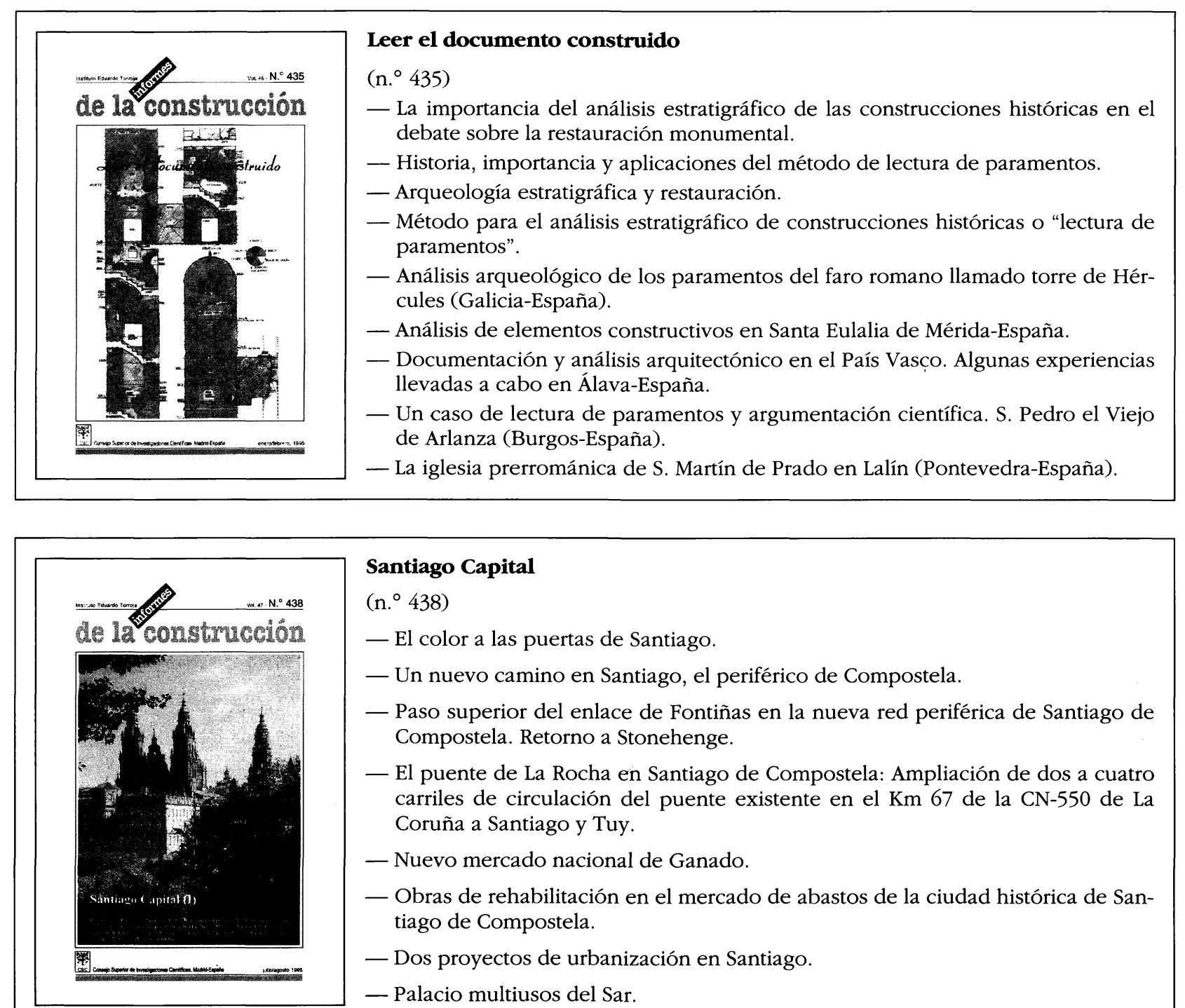

\section{Santiago Capital}

(n. $\left.{ }^{\circ} 438\right)$

- El color a las puertas de Santiago.

- Un nuevo camino en Santiago, el periférico de Compostela.

- Paso superior del enlace de Fontiñas en la nueva red periférica de Santiago de Compostela. Retorno a Stonehenge.

- El puente de La Rocha en Santiago de Compostela: Ampliación de dos a cuatro carriles de circulación del puente existente en el $\mathrm{Km} 67$ de la $\mathrm{CN}-550$ de La Coruña a Santiago y Tuy.

- Nuevo mercado nacional de Ganado.

- Obras de rehabilitación en el mercado de abastos de la ciudad histórica de Santiago de Compostela.

- Dos proyectos de urbanización en Santiago.

- Palacio multiusos del Sar.

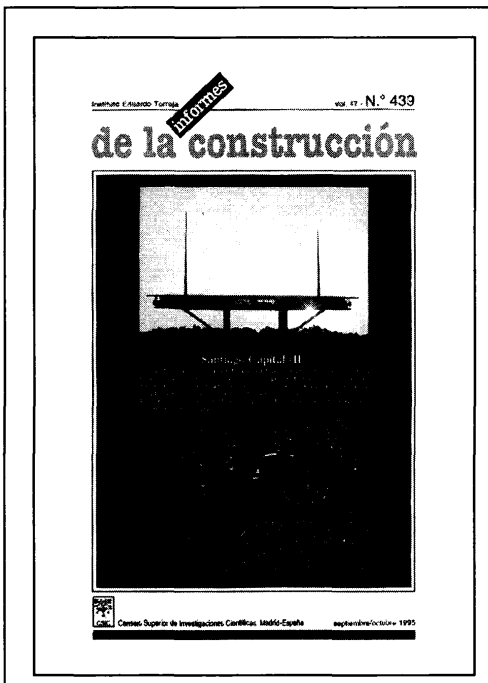

(n. $\left.{ }^{\circ} 439\right)$

- La transformación urbanística de Compostela. El planeamiento urbano al servicio de un proyecto de desarrollo (Galicia-España).

- Palacio de Congresos y Exposiciones de Galicia en Santiago (Galicia-España).

- Nueva instalación de Telecomunicaciones. Santiago de Compostela (Galicia-España).

- Centro de Arte Contemporáneo de Galicia en Santiago de Compostela (Galicia-España).

— Escuela Pública "Carme de Abaixo". Santiago de Compostela (Galicia-España).

- Avenida Xoan XXIII. Santiago de Compostela (Galicia-España).

- Edificios para Instituto de Investigación de la Universidad de Santiago. Santiago de Compostela (Galicia-España).

— La Oficina Municipal de Conservación y Rehabilitación de la Ciudad Histónca de Santiago de Compostela (Galicia-España). 